\title{
RELATIONS BETWEEN ABS-NORMAL NLPS AND MPCCS. PART 1: STRONG CONSTRAINT QUALIFICATIONS
}

\author{
L.C. Hegerhorst-Schultchen ${ }^{*}$ \\ C. Kirches ${ }^{\dagger}$ \\ M.C. Steinbach ${ }^{\ddagger}$
}

\begin{abstract}
This work is part of an ongoing effort of comparing non-smooth optimization problems in abs-normal form to MPCCs. We study the general abs-normal NLP with equality and inequality constraints in relation to an equivalent MPCC reformulation. We show that kink qualifications and MPCC constraint qualifications of linear independence type and Mangasarian-Fromovitz type are equivalent. Then we consider strong stationarity concepts with first and second order optimality conditions, which again turn out to be equivalent for the two problem classes. Throughout we also consider specific slack reformulations suggested in [9], which preserve constraint qualifications of linear independence type but not of Mangasarian-Fromovitz type.
\end{abstract}

\section{INTRODUCTION}

Nonsmoothness arises frequently in practical optimization problems from various areas. In finite dimensions, certain nonsmooth functions like $\ell_{1}$ and $\ell_{\infty}$ norms can be avoided by smooth remodeling, but models that involve (possibly nested) absolute value, maximum, and minimum functions, models with piecewise definitions and models with equilibrium conditions or complementarity conditions lead to genuine nonsmoothness. An important sub-class of such nonsmooth finite-dimensional optimization problems is essentially characterized as "NLPs with finitely many kinks", which gives rise to more general standard problem classes like MPCCs [10] and optimization problems in abs-normal form [2].

The latter are non-smooth nonlinear optimization problems of the form

$$
\begin{array}{lll}
\min _{x} f(x) \quad \text { s.t. } & g(x)=0 \\
& h(x) \geq 0
\end{array}
$$

where $D^{x} \subseteq \mathbb{R}^{n}$ is open, the objective $f \in C^{d}\left(D^{x}, \mathbb{R}\right)$ is a smooth function $(d \geq 1)$ and the equality and inequality constraints $g \in C_{\text {abs }}^{d}\left(D^{x}, \mathbb{R}^{m_{1}}\right)$ and $h \in C_{\text {abs }}^{d}\left(D^{x}, \mathbb{R}^{m_{2}}\right)$ are non-smooth functions with the non-smoothness exposed in abs-normal form [2]. Thus, there exist functions $c_{\mathcal{E}} \in C^{d}\left(D^{x,|z|}, \mathbb{R}^{m_{1}}\right)$, $c_{I} \in C^{d}\left(D^{x,|z|}, \mathbb{R}^{m_{2}}\right)$ and $c_{\mathcal{Z}} \in C^{d}\left(D^{x,|z|}, \mathbb{R}^{s}\right)$ with $D^{x,|z|}=D^{x} \times D^{|z|}, D^{|z|} \subseteq \mathbb{R}^{s}$ open and symmetric

C. Kirches discloses financial support by the German Federal Ministry of Education and Research through grants $\mathrm{n}^{\mathrm{O}}$ o5M17MBA-MoPhaPro, o5M18MBA-MOReNet, 01/S17089C-ODINE, and o5M20MBA-LEOPLAN, and by Deutsche Forschungsgemeinschaft (DFG) through Priority Programme 1962, grant Ki1839/1-2.

*Leibniz Universität Hannover, Institut für Angewandte Mathematik, Welfengarten 1, 30167 Hannover, Germany. hegerhorst@ifam.uni-hannover.de

$\dagger$ Technische Universität Carolo-Wilhelmina zu Braunschweig, Institut für Mathematische Optimierung, Universitätsplatz 2, 38106 Braunschweig, Germany. c.kirches@tu-bs.de

¥Leibniz Universität Hannover, Institut für Angewandte Mathematik, Welfengarten 1, 30167 Hannover, Germany. mcs@ifam.uni-hannover.de 
(i.e., $z \in D^{|z|}$ implies $\Sigma z \in D^{|z|}$ for every signature matrix $\Sigma$, see Definition 1.1) such that

$$
\begin{aligned}
g(x) & =c_{\mathcal{E}}(x,|z|), \\
h(x) & =c_{\mathcal{I}}(x,|z|), \\
z & =c_{\mathcal{Z}}(x,|z|) \quad \text { with } \partial_{2} c_{\mathcal{Z}}(x,|z|) \text { strictly lower triangular. }
\end{aligned}
$$

Note that we introduce one joint switching constraint $c_{\mathcal{Z}}$ for $g$ and $h$ and reuse switching variables $z_{i}$ if the same argument occurs inside an absolute value in $g$ and $h$. Here, components of $z$ can be computed one by one from $x$ and $z_{i}, i<j$, since $\partial_{2} c_{\mathcal{Z}}(x,|z|)$ is strictly lower triangular. In the following we write $z(x)$ to denote this dependence explicitly. However, $z$ is implicitly defined by $z=c_{\mathcal{Z}}(x,|z|)$. To consider solvability of this system, we use the reformulation $\left|z_{i}\right|=\operatorname{sign}\left(z_{i}\right) z_{i}$.

Definition 1.1 (Signature of $z$ ). Let $x \in D^{x}$. We define the signature $\sigma(x)$ and the associated signature matrix $\Sigma(x)$ as

$$
\sigma(x):=\operatorname{sign}(z(x)) \in\{-1,0,1\}^{s}, \quad \Sigma(x):=\operatorname{diag}(\sigma(x)) .
$$

A signature vector $\sigma(x) \in\{-1,1\}^{s}$ is called definite, otherwise indefinite.

With Definition 1.1, we can write $|z(x)|=\Sigma(x) z(x)$ and consider the system $z=c_{\mathcal{Z}}(x, \Sigma z)$, which for any fixed signature $\Sigma=\Sigma(\hat{x})$ becomes a differentiable system. Then, application of the implicit function theorem yields the existence of a locally unique solution $z(x)$ with Jacobian

$$
\partial_{x} z(\hat{x})=\left[I-\partial_{2} c_{\mathcal{Z}}(\hat{x},|z(\hat{x})|) \Sigma\right]^{-1} \partial_{1} c_{\mathcal{Z}}(\hat{x},|z(\hat{x})|) \in \mathbb{R}^{s \times n} .
$$

Definition 1.2 (Active Switching Set). We call the switching variable $z_{i}$ active if $z_{i}(x)=0$. The active switching set $\alpha$ consists of all indices of active switching variables, i.e.

$$
\alpha(x):=\left\{i \in\{1, \ldots, s\}: z_{i}(x)=0\right\} .
$$

We denote the number of active switching variables by $|\alpha(x)|$ and the number of inactive ones by $|\sigma(x)|:=s-|\alpha(x)|$.

Unconstrained optimization problems in abs-normal form have recently been introduced by [3], and have been shown to come with favorable theoretical properties that lead to globally convergent solution methods based on piecewise linearizations that can be generated by algorithmic differentiation $[5,6]$. When nonsmooth equality and inequality constraints are added, the resulting abs-normal NLPs can be compared to MPCCs, for which a well-established theoretical framework has been developed over the past decades [10]. Comparing the two classes of nonsmooth optimization problems, the authors reported in [8] that they basically form the same problem class, although with a different representation of nonsmoothness and not necessarily with the same regularity properties.

\section{LITERATURE}

The abs-normal NLPs considered here are a direct generalization of unconstrained abs-normal problems developed by Griewank and Walther [2, 3]. These problems offer particularly attractive theoretical features when generalizing KKT theory and stationarity concepts, and they are tractable by sophisticated algorithms with guaranteed convergence based on piecewise linearizations and using algorithmic differentiation techniques $[5,6]$.

Another important class of nonsmooth optimization problems are Mathematical Programs with Complementarity (or Equilibrium) Constraints (MPCCs, MPECs); an overview can be found in the book [10]. Since standard theory for smooth optimization problems cannot be applied, new constraint qualifications and corresponding optimality conditions were introduced. By now, there is a large body 
of literature on MPCCs, and we refer to [12] for an overview of the basic concepts and theory. In this paper, constraint qualifications for MPCCs in the sense of linear independence and Mangasarian Fromowitz are considered. Further, the corresponding stationarity concept (S-stationarity) as well as first and second order optimality conditions are studied. Details can be found in [11], [10] and [1].

In [8] we have shown that unconstrained optimization problems in abs-normal form are a subclass of MPCCs and we have studied regularity concepts of linear independence type, Mangasarian-Fromovitz type and Abadie type. We have also shown that abs-normal NLPs with general constraints are equivalent to the class of MPCCs. In [9] we have generalized optimality conditions of unconstrained abs-normal problems to the case with equality and inequality constraints under the linear independence kink qualification. More details and additional information about these results as well as about the results in this paper can be found in [7].

\section{CONTRIBUTIONS}

We develop a deeper understanding of the commonalities of these problem classes of NLPs in absnormal form on the one hand, and MPCCs on the other. We provide a detailed comparative study of general abs-normal NLPs and MPCCs, considering constraint qualifications of linear independence type and Mangasarian Fromovitz type for the standard formulation and for a reformulation with absolute value slacks that was suggested in [9]. In particular, we show that corresponding constraint qualifications of abs-normal NLPs and MPCCs are equivalent and that the linear independence type constraint qualifications are preserved by the slack reformulation while this is not the case for a Mangasarian-Fromovitz type constraint qualification. We then compare optimality conditions of first and second order for abs-normal NLPs and MPCCs under the respective linear independence type constraint qualifications. We show equivalence of the respective first order necessary conditions, kink stationarity and strong stationarity. We also show how second order conditions for MPCCs can be carried over to abs-normal NLPs. Under suitable additional assumptions, we prove equivalence of positive (semi-)definiteness of the associated reduced Hessians, which gives correspondences of second order necessary and sufficient conditions.

We expect that our theoretical results will contribute to the understanding and further development of rigorous solution algorithms for abs-normal NLPs. We also expect the results to facilitate a possible transferral of active-signature methods for abs-normal forms, such as SALMIN [5], to MPCCs.

\section{STRUCTURE}

The remainder of this article is structured as follows. In Section 2 we present the general abs-normal NLP and its slack reformulation, and we formulate the associated kink qualifications and compare them. In Section 3 we introduce counterpart MPCCs for the two formulations of abs-normal NLPs and compare the associated MPCC-constraint qualifications. Then, we show equivalence of the regularity concepts for abs-normal NLPs and MPCCs in Section 4. Finally, in Section 5 we state optimality conditions of first and second-order for abs-normal NLPs and MPCCs and prove equivalence relations between them. We conclude in Section 6 and give a brief outlook.

\section{ABS-NORMAL NLPS}

In this section we consider two formulations for non-smooth NLPs in abs-normal form that differ in the treatment of inequality constraints. 


\subsection{GENERAL ABS-NORMAL NLPS}

In this paragraph we consider abs-normal NLPs with equalities and inequalities, obtained by substituting the constraints representation in abs-normal form (ANF) into the general non-smooth problem (NLP). Note that we use the variables $\left(t, z^{t}\right)$ instead of $(x, z)$.

Definition 2.1 (Abs-Normal NLP). Let $D^{t}$ be an open subset of $\mathbb{R}^{n_{t}}$. A non-smooth NLP is called an abs-normal NLP if functions $f \in C^{d}\left(D^{t}, \mathbb{R}\right), c_{\mathcal{E}} \in C^{d}\left(D^{t,\left|z^{t}\right|}, \mathbb{R}^{m_{1}}\right), c_{\mathcal{I}} \in C^{d}\left(D^{t,\left|z^{t}\right|}, \mathbb{R}^{m_{2}}\right)$, and $c_{\mathcal{Z}} \in C^{d}\left(D^{t,\left|z^{t}\right|}, \mathbb{R}^{s_{t}}\right)$ with $d \geq 1$ exist such that the NLP reads

$(\mathrm{I}-\mathrm{NLP})$

$$
\begin{aligned}
\min _{t, z^{t}} f(t) \quad \text { s.t. } & c_{\mathcal{E}}\left(t,\left|z^{t}\right|\right)=0, \\
& c_{\mathcal{I}}\left(t,\left|z^{t}\right|\right) \geq 0, \\
& c_{\mathcal{Z}}\left(t,\left|z^{t}\right|\right)-z^{t}=0,
\end{aligned}
$$

where $D^{\left|z^{t}\right|}$ is open and symmetric and $\partial_{2} c_{\mathcal{Z}}\left(x,\left|z^{t}\right|\right)$ is strictly lower triangular.

The feasible set of (I-NLP) is denoted by

$$
\begin{aligned}
\mathcal{F}_{\text {abs }} & :=\left\{\begin{array}{l|l}
\left(t, z^{t}\right) & \begin{array}{l}
c_{\mathcal{E}}\left(t,\left|z^{t}\right|\right)=0, c_{\mathcal{I}}\left(t,\left|z^{t}\right|\right) \geq 0, \\
c_{\mathcal{Z}}\left(t,\left|z^{t}\right|\right)-z^{t}=0
\end{array}
\end{array}\right\} \\
& =\left\{\left(t, z^{t}(t)\right): t \in D^{t}, c_{\mathcal{E}}\left(t,\left|z^{t}(t)\right|\right)=0, c_{\mathcal{I}}\left(t,\left|z^{t}(t)\right|\right) \geq 0\right\} .
\end{aligned}
$$

In contrast to standard NLP theory, we do not count equalities as active constraints.

Definition 2.2 (Active Inequality Set). Let $\left(t, z^{t}(t)\right) \in \mathcal{F}_{\text {abs. }}$. We call the constraint $i \in \mathcal{I}$ active if $c_{i}\left(t,\left|z^{t}(t)\right|\right)=0$. The active set $\mathcal{A}(t)$ consists of all indices of active constraints,

$$
\mathcal{A}(t)=\left\{i \in \mathcal{I}: c_{i}\left(t,\left|z^{t}(t)\right|\right)=0\right\} .
$$

We denote the number of active inequality constraints by $|\mathcal{A}(t)|$.

To define the linear independence kink qualification as well as the interior direction kink qualification for (I-NLP) we need its Jacobians.

Definition 2.3 (Jacobians). Consider the abs-normal NLP (I-NLP). For $\left(t, z^{t}(t)\right) \in \mathcal{F}_{\text {abs }}$ set $\mathcal{A}=\mathcal{A}(t)$, $\alpha=\alpha(t), \sigma=\sigma(t), \Sigma=\operatorname{diag}(\sigma)$, and $c_{\mathcal{A}}=\left[c_{i}\right]_{i \in \mathcal{A}}$. The equality-constraints facobian is

$$
\begin{aligned}
J_{\mathcal{E}}(t):=\partial_{t} c_{\mathcal{E}}\left(t, \Sigma z^{t}(t)\right) & =\partial_{1} c_{\mathcal{E}}\left(t, \Sigma z^{t}(t)\right)+\partial_{2} c_{\mathcal{E}}\left(t, \Sigma z^{t}(t)\right) \sum \partial_{t} z^{t}(t) \\
& =\partial_{1} c_{\mathcal{E}}\left(t,\left|z^{t}(t)\right|\right)+\partial_{2} c_{\mathcal{E}}\left(t,\left|z^{t}(t)\right|\right) \sum \partial_{t} z^{t}(t)
\end{aligned}
$$

the active inequality facobian is

$$
\begin{aligned}
J_{\mathcal{A}}(t):=\partial_{t} c_{\mathcal{A}}\left(t, \Sigma z^{t}(t)\right) & =\partial_{1} c_{\mathcal{A}}\left(t, \Sigma z^{t}(t)\right)+\partial_{2} c_{\mathcal{A}}\left(t, \Sigma z^{t}(t)\right) \Sigma \partial_{t} z^{t}(t) \\
& =\partial_{1} c_{\mathcal{A}}\left(t,\left|z^{t}(t)\right|\right)+\partial_{2} c_{\mathcal{A}}\left(t,\left|z^{t}(t)\right|\right) \Sigma \partial_{t} z^{t}(t),
\end{aligned}
$$

and the active switching facobian is

$$
J_{\alpha}(t):=\left[e_{i}^{T} \partial_{t} z^{t}(t)\right]_{i \in \alpha}=\left[e_{i}^{T}\left[I-\partial_{2} c \mathcal{Z}\left(t,\left|z^{t}(t)\right|\right) \Sigma\right]^{-1} \partial_{1} c_{\mathcal{Z}}\left(t,\left|z^{t}(t)\right|\right)\right]_{i \in \alpha} .
$$

Definition 2.4 (Linear Independence Kink Qualification). Let $\left(t, z^{t}(t)\right) \in \mathcal{F}_{\text {abs. }}$. We say that the linear independence kink qualification (LIKQ) holds for (I-NLP) at $t$ if

$$
J_{\text {abs }}(t)=\left[\begin{array}{c}
J_{\mathcal{E}}(t) \\
J_{\mathcal{A}}(t) \\
J_{\alpha}(t)
\end{array}\right]=\left[\begin{array}{c}
\partial_{t} c_{\mathcal{E}}\left(t,\left|z^{t}(t)\right|\right) \\
\partial_{t} c_{\mathcal{A}}\left(t,\left|z^{t}(t)\right|\right) \\
{\left[e_{i}^{T} \partial_{t} z^{t}(t)\right]_{i \in \alpha}}
\end{array}\right] \in \mathbb{R}^{\left(m_{1}+|\mathcal{A}|+|\alpha|\right) \times n_{t}}
$$

has full row rank $m_{1}+|\mathcal{A}|+|\alpha|$. 
Definition 2.5 (Interior Direction Kink Qualification). Let $\left(t, z^{t}(t)\right) \in \mathcal{F}_{\text {abs }}$. We say that the interior direction kink qualification (IDKQ) holds for (I-NLP) at $t$ if

$$
\left[\begin{array}{l}
J_{\mathcal{E}}(t) \\
J_{\alpha}(t)
\end{array}\right]=\left[\begin{array}{l}
\partial_{t} c_{\mathcal{E}}\left(t,\left|z^{t}(t)\right|\right) \\
{\left[e_{i}^{T} \partial_{t} z^{t}(t)\right]_{i \in \alpha}}
\end{array}\right] \in \mathbb{R}^{\left(m_{1}+|\alpha|\right) \times n_{t}}
$$

has full row rank $m_{1}+|\alpha|$ and if there exists a vector $d \in \mathbb{R}^{n_{t}}$ such that

$$
J_{\mathcal{E}}(t) d=0, \quad J_{\alpha}(t) d=0, \quad \text { and } \quad J_{\mathcal{A}}(t) d>0 .
$$

For the general abs-normal NLP (I-NLP) considered here, IDKQ actually generalizes MFCQ from the smooth case and corresponds to MPCC-MFCQ, as we will show below. We cannot use the canonical name MFKQ, however, since Griewank and Walther have already defined MFKQ as a different weakening of LIKQ in [4]. We believe that other possible names like "Abs-normal MFKQ" or "Constrained MFKQ" would produce confusion rather than clarification and hence suggest the descriptive name "Interior Direction KQ".

The following example from [11] (converted from MPCC form to abs-normal NLP form) shows that IDKQ is weaker than LIKQ in the presence of inequality constraints.

Example 2.6 (IDKQ is weaker than LIKQ). Consider the problem

$$
\begin{aligned}
& \min _{t \in \mathbb{R}^{3}, z^{t} \in \mathbb{R}} t_{1}+t_{2}-t_{3} \quad \text { s.t. } \quad t_{1}+t_{2}-\left|z^{t}\right|=0, \\
& 4 t_{1}-t_{3} \geq 0 \text {, } \\
& 4 t_{2}-t_{3} \geq 0 \text {, } \\
& t_{1}-t_{2}-z^{t}=0 \text {, }
\end{aligned}
$$

with solution $t^{*}=(0,0,0)$ and $\left(z^{t}\right)^{*}=0$. We compute

$$
J_{\mathcal{A}}\left(t^{*}\right)=\left[\begin{array}{ccc}
4 & 0 & -1 \\
0 & 4 & -1
\end{array}\right], \quad J_{\mathcal{E}}\left(t^{*}\right)=\left[\begin{array}{lll}
1 & 1 & 0
\end{array}\right], \quad \text { and } \quad J_{\alpha}\left(t^{*}\right)=\left[\begin{array}{lll}
1 & -1 & 0
\end{array}\right] .
$$

Here, LIKQ is not satisfied but IDKQ is satisfied with $d=(0,0,-1)$.

\subsection{ABS-NORMAL NLPS WITH INEQUALITY SLACKS}

In this paragraph we consider abs-normal NLPs with slack variables introduced for all inequalities. We make use of the absolute value of a slack variable, an idea due to Griewank. This results in a class of purely equality-constrained abs-normal NLPs, which simplifies the derivation of optimality conditions under the LIKQ, see [9] and Section 5.

Using slack variables $w \in \mathbb{R}^{m_{2}}$, we obtain the following reformulation of (NLP):

$$
\begin{array}{ll}
\min _{t, w} f(t) \quad \text { s.t. } & g(t)=0, \\
& h(t)-|w|=0 .
\end{array}
$$

Then, we express $g$ and $h$ in abs-normal form as in (ANF) and introduce additional switching variables $z^{w}$ to handle $|w|$. This approach leads to the next definition.

Definition 2.7 (Abs-Normal NLP with Inequality Slacks). An abs-normal NLP posed in the following form is called an abs-normal NLP with inequality slacks:

$$
\begin{aligned}
\min _{t, w, z^{t}, z^{w}} f(t) \quad \text { s.t. } & c_{\mathcal{E}}\left(t,\left|z^{t}\right|\right)=0, \\
& c_{\mathcal{I}}\left(t,\left|z^{t}\right|\right)-\left|z^{w}\right|=0, \\
& c_{\mathcal{Z}}\left(t,\left|z^{t}\right|\right)-z^{t}=0, \\
& w-z^{w}=0,
\end{aligned}
$$

where $D^{\left|z^{t}\right|}$ is open and symmetric and $\partial_{2} c_{\mathcal{Z}}\left(x,\left|z^{t}\right|\right)$ is strictly lower triangular. 
The feasible set of (E-NLP) is a lifting of $\mathcal{F}_{\text {abs }}$,

$$
\begin{aligned}
\mathcal{F}_{\text {e-abs }} & :=\left\{\begin{array}{l|l}
\left(t, w, z^{t}, z^{w}\right) & \begin{array}{l}
c_{\mathcal{E}}\left(t,\left|z^{t}\right|\right)=0, c_{I}\left(t,\left|z^{t}\right|\right)-\left|z^{w}\right|=0, \\
c_{\mathcal{Z}}\left(t,\left|z^{t}\right|\right)-z^{t}=0, w-z^{w}=0
\end{array}
\end{array}\right\} \\
& =\left\{\left(t, w, z^{t}, z^{w}\right):\left(t, z^{t}\right) \in \mathcal{F}_{\mathrm{abs}}, w=z^{w},\left|z^{w}\right|=c_{I}\left(t,\left|z^{t}\right|\right)\right\} .
\end{aligned}
$$

Using the dependence of $z^{t}$ and $z^{w}$ of $t$ and $w$, the feasible set can be written as

$$
\begin{aligned}
\mathcal{F}_{\text {e-abs }} & =\left\{\begin{array}{l|l}
\left(t, w, z^{t}(t), z^{w}(w)\right) \mid \begin{array}{l}
t \in D^{t}, c_{\mathcal{E}}\left(t,\left|z^{t}(t)\right|\right)=0, \\
c_{I}\left(t,\left|z^{t}(t)\right|\right)-\left|z^{w}(w)\right|=0, w-z^{w}(w)=0
\end{array}
\end{array}\right\} \\
& =\left\{\left(t, w, z^{t}(t), z^{w}(w)\right):\left(t, z^{t}(t)\right) \in \mathcal{F}_{\mathrm{abs}}, w=z^{w}(w),\left|z^{w}(w)\right|=c_{I}\left(t,\left|z^{t}(t)\right|\right)\right\} .
\end{aligned}
$$

We split the active switching set into subsets for variables $t$ and $w$ as $\alpha=\left(\alpha^{t}, \alpha^{w}\right)$.

Remark 2.8. Note that introducing $|w|$ converts inequalities to pure equalities without a nonnegativity condition for the slack variables $w$. However, the slack reformulation has some subtle issues. Subsequently we will show that, in contrast to LIKQ, IDKQ is not preserved. Moreover, one cannot eliminate the equation $w-z^{w}=0$ (and hence $z^{w}$ or $w$ ) in (E-NLP) since this would destroy the abs-normal form. Finally, the slack $w$ is not uniquely determined since the signs of nonzero components $w_{i}$ can be chosen arbitrarily, yielding a set of $2^{m_{2}-\left|\alpha^{w}\right|}$ choices, $W(t):=\left\{w:|w|=c_{I}\left(t,\left|z^{t}(t)\right|\right)\right\}$.

Lemma 2.9. Consider $\left(t, w, z^{t}(t), z^{w}(w)\right) \in \mathcal{F}_{\mathrm{e}-\mathrm{abs}}$. Then, LIKQ for (E-NLP) at $(t, w)$ is full row rank of

$$
J_{\text {e-abs }}(t, w)=\left[\begin{array}{cc}
\partial_{t} c_{\mathcal{E}}\left(t,\left|z^{t}(t)\right|\right) & 0 \\
\partial_{t} c_{I}\left(t,\left|z^{t}(t)\right|\right) & -\Sigma^{w} \\
{\left[e_{i}^{T} \partial_{t} z^{t}(t)\right]_{i \in \alpha^{t}}} & 0 \\
0 & {\left[e_{i}^{T} I\right]_{i \in \alpha^{w}}}
\end{array}\right] \in \mathbb{R}^{\left(m_{1}+m_{2}+\left|\alpha^{t}\right|+\left|\alpha^{w}\right|\right) \times\left(n_{t}+m_{2}\right)} .
$$

Proof. Set $x=(t, w), z=\left(z^{t}, z^{w}\right), \bar{f}(x)=f(t), \bar{c}_{\mathcal{E}}(x,|z|)=\left(c_{\mathcal{E}}\left(t,\left|z^{t}\right|\right), c_{\mathcal{I}}\left(t,\left|z^{t}\right|\right)-\left|z^{w}\right|\right)$, and $\bar{c}_{\mathcal{Z}}(x,|z|)=\left(c_{\mathcal{Z}}\left(t,\left|z^{t}\right|\right), w\right)$. Then, we can write (E-NLP) compactly as

$(\overline{\mathrm{E}-\mathrm{NLP}})$

$$
\begin{aligned}
\min _{x, z} \bar{f}(x) \quad \text { s.t. } \quad & \bar{c}_{\mathcal{E}}(x,|z|)=0, \\
& \bar{c}_{\mathcal{Z}}(x,|z|)-z=0,
\end{aligned}
$$

and compute $\bar{J}_{\mathcal{E}}$ and $\bar{J}_{\alpha}$ from Definition 2.3 using the special structure of (E-NLP). The resulting matrix $J_{\mathrm{e}-\mathrm{abs}}(x)=\left[\begin{array}{ll}\bar{J}_{\mathcal{E}}(x)^{T} & \bar{J}_{\alpha}(x)^{T}\end{array}\right]^{T}$ in Definition 2.4 has the form above.

Remark 2.10. Clearly, the rank of $J_{\mathrm{e} \text {-abs }}$ does not depend on the signs of \pm 1 entries in $\Sigma^{w}$ but only on their positions. Hence, LIKQ does not depend on the particular choice of $w$. Otherwise it would not make sense to consider (E-NLP).

Note that, since the abs-normal NLP (E-NLP) does not contain any inequalities, the concept of IDKQ is equivalent to LIKQ here. This is in contrast to the standard reformulation of smooth NLP inequalities as equalities with nonnegative slacks where the validity of LICQ and MFCQ are both unaffected.

\subsection{RELATIONS OF KINK QUALIFICATIONS FOR ABS-NORMAL NLPS}

In this paragraph we discuss the relations of kink qualifications for the two different formulations of abs-normal NLPs. We use the set $W(t)$ from above.

Theorem 2.11. LIKQ for (I-NLP) holds at $\left(t, z^{t}(t)\right) \in \mathcal{F}_{\text {abs }}$ if and only if LIKQ for (E-NLP) holds at $\left(t, w, z^{t}(t), z^{w}(w)\right) \in \mathcal{F}_{\mathrm{e}-\mathrm{abs}}$ for any (and hence all) $w \in W(t)$. 
Proof. This follows immediately by comparison of $J_{\mathrm{abs}}$ and $J_{\mathrm{e}-\mathrm{abs}}$ using that

$$
\alpha^{w}(w)=\left\{i \in \mathcal{I}: w_{i}=0\right\}=\left\{i \in \mathcal{I}: c_{i}\left(t, z^{t}(t)\right)=0\right\}=\mathcal{A}(t)
$$

and

$$
\Sigma^{w}=\operatorname{diag}\left(\sigma^{w}\right) \quad \text { with } \quad \sigma_{i}^{w}=\operatorname{sign}\left(w_{i}\right)= \begin{cases}0, & i \in \mathcal{A}(t), \\ \pm 1, & i \notin \mathcal{A}(t) .\end{cases}
$$

Theorem 2.12. IDKQ for (I-NLP) holds at $\left(t, z^{t}(t)\right) \in \mathcal{F}_{\text {abs }}$ if IDKQ for (E-NLP) holds at the lifted point $\left(t, w, z^{t}(t), z^{w}(w)\right) \in \mathcal{F}_{\mathrm{e}-\text { abs }}$ for any (and hence all) $w \in W(t)$. The converse is not true.

Proof. Since (E-NLP) has no inequalities, the concepts of IDKQ and LIKQ coincide. LIKQ for (E-NLP) is equivalent to LIKQ for (I-NLP) by Theorem 2.11, and LIKQ for (I-NLP) implies IDKQ for (I-NLP). The converse does not hold since LIKQ for (I-NLP) is stronger then IDKQ as we have shown in Example 2.6.

\section{COUNTERPART MPCCS}

In this section we introduce MPCC counterpart problems for the two formulations (I-NLP) and (E-NLP). Then, we have a quick look at relations between them.

\subsection{COUNTERPART MPCC FOR THE GENERAL ABS-NORMAL NLP}

To reformulate (I-NLP) as an MPCC, we partition $z^{t}$ into its nonnegative part and the modulus of its nonpositive part, $u^{t}:=\left[z^{t}\right]^{+}:=\max \left(z^{t}, 0\right)$ and $v^{t}:=\left[z^{t}\right]^{-}:=\max \left(-z^{t}, 0\right)$. Then, we require complementarity of these two variables to replace $\left|z^{t}\right|$ by $u^{t}+v^{t}$ and $z^{t}$ itself by $u^{t}-v^{t}$.

Definition 3.1 (Counterpart MPCC of (I-NLP)). The counterpart MPCC of the abs-normal NLP (I-NLP) reads

(I-MPCC)

$$
\begin{aligned}
\min _{t, u^{t}, v^{t}} f(t) \quad \text { s.t. } \quad & c_{\mathcal{E}}\left(t, u^{t}+v^{t}\right)=0, \\
& c_{I}\left(t, u^{t}+v^{t}\right) \geq 0, \\
& c_{\mathcal{Z}}\left(t, u^{t}+v^{t}\right)-\left(u^{t}-v^{t}\right)=0, \\
& 0 \leq u^{t} \perp v^{t} \geq 0,
\end{aligned}
$$

where $u^{t}, v^{t} \in \mathbb{R}^{s_{t}}$.

The feasible set of (I-MPCC) is denoted by

$$
\mathcal{F}_{\text {mpcc }}:=\left\{\begin{array}{l|l}
\left(t, u^{t}, v^{t}\right) & \begin{array}{l}
c_{\mathcal{E}}\left(t, u^{t}+v^{t}\right)=0, c_{I}\left(t, u^{t}+v^{t}\right) \geq 0 \\
c_{\mathcal{Z}}\left(t, u^{t}+v^{t}\right)=u^{t}-v^{t}, 0 \leq u^{t} \perp v^{t} \geq 0
\end{array}
\end{array}\right\} .
$$

Lemma 3.2. Given an abs-normal NLP (I-NLP) and its counterpart MPCC (I-MPCC), we have a homeomorphism $\phi: \mathcal{F}_{\text {mpcc }} \rightarrow \mathcal{F}_{\text {abs }}$ defined as

$$
\phi\left(t, u^{t}, v^{t}\right)=\left(t, u^{t}-v^{t}\right), \quad \phi^{-1}\left(t, z^{t}\right)=\left(t,\left[z^{t}\right]^{+},\left[z^{t}\right]^{-}\right) .
$$

Proof. Obvious. 
Just like the active switching set of the abs-normal NLP, we define index sets of the counterpart MPCC.

Definition 3.3 (Index Sets). We denote by $\mathcal{U}_{0}^{t}:=\left\{i \in\left\{1, \ldots, s_{t}\right\}: u_{i}^{t}=0\right\}$ the set of indices of active inequalities $u_{i}^{t} \geq 0$, and by $\mathcal{U}_{+}^{t}:=\left\{i \in\left\{1, \ldots, s_{t}\right\}: u_{i}^{t}>0\right\}$ the set of indices of inactive inequalities $u_{i}^{t} \geq 0$. Analogous definitions hold of $\mathcal{V}_{0}^{t}$ and $\mathcal{V}_{+}^{t}$. By $\mathcal{D}^{t}:=\mathcal{U}_{0}^{t} \cap \mathcal{V}_{0}^{t}$ we denote the set of indices of nonstrict (degenerate) complementarity pairs. Thus we have the partitioning $\left\{1, \ldots, s_{t}\right\}=\mathcal{U}_{+}^{t} \cup \mathcal{V}_{+}^{t} \cup \mathcal{D}^{t}$.

In the following we define constraint qualifications for the counterpart MPCC. The standard definitions say that MPCC-LICQ and MPCC-MFCQ are LICQ and MFCQ, respectively, for the so-called tightened NLP (see [11]) with associated Jacobian

$$
J\left(t, u^{t}, v^{t}\right)=\left[\begin{array}{ccccc}
\partial_{1} c_{\mathcal{E}} & \partial_{2} c_{\mathcal{E}} P_{\mathcal{U}_{+}^{t}}^{T} & \partial_{2} c_{\mathcal{E}} P_{\mathcal{U}_{0}^{t}}^{T} & \partial_{2} c_{\mathcal{E}} P_{\mathcal{V}_{+}^{t}}^{T} & \partial_{2} c_{\mathcal{E}} P_{\mathcal{V}_{0}^{t}}^{T} \\
\partial_{1} c_{\mathcal{A}} & \partial_{2} c_{\mathcal{A}} P_{\mathcal{U}_{+}^{t}}^{T} & \partial_{2} c_{\mathcal{A}} P_{\mathcal{U}_{0}^{t}}^{T} & \partial_{2} c_{\mathcal{A}} P_{\mathcal{V}_{+}^{t}}^{T} & \partial_{2} c_{\mathcal{A}} P_{\mathcal{V}_{0}^{t}}^{T} \\
\partial_{1} c_{\mathcal{Z}} & {\left[\partial_{2} c_{\mathcal{Z}}-I\right] P_{\mathcal{U}_{+}^{t}}^{T}} & {\left[\partial_{2} c_{\mathcal{Z}}-I\right] P_{\mathcal{U}_{0}^{t}}^{T}} & {\left[\partial_{2} c_{\mathcal{Z}}+I\right] P_{\mathcal{V}_{+}^{t}}^{T}} & {\left[\partial_{2} c_{\mathcal{Z}}+I\right] P_{\mathcal{V}_{0}^{t}}^{T}} \\
0 & 0 & I & 0 & 0 \\
0 & 0 & 0 & 0 & I
\end{array}\right],
$$

where $P_{\mathcal{S}} \in \mathbb{R}^{|\mathcal{S}| \times s_{t}}$ denotes the projector onto the subspace defined by $\mathcal{S} \subseteq\left\{1, \ldots, s_{t}\right\}$ and all partial darivatives are evaluated at $\left(t, u^{t}+v^{t}\right)$. This Jacobian will be needed in Section 5.2 to formulate second order conditions. Here we exploit the two unit blocks to state constraint qualifications in a more compact form as in [8].

Definition 3.4 (MPCC-LICQ for (I-MPCC), see [11]). We say that the MPCC-LICQ holds for (I-MPCC) at a feasible point $\left(t, u^{t}, v^{t}\right)$ if

$$
\begin{aligned}
J_{\text {mpcc }}\left(t, u^{t}, v^{t}\right) & =\left[\begin{array}{ccc}
\partial_{1} c_{\mathcal{E}} & \partial_{2} c_{\mathcal{E}} P_{\mathcal{U}_{+}^{t}}^{T} & \partial_{2} c_{\mathcal{E}} P_{\mathcal{V}_{+}^{t}}^{T} \\
\partial_{1} c_{\mathcal{A}} & \partial_{2} c_{\mathcal{A}} P_{\mathcal{U}_{+}^{t}}^{T} & \partial_{2} c_{\mathcal{A}} P_{\mathcal{V}_{+}^{t}}^{T} \\
\partial_{1} c_{\mathcal{Z}} & {\left[\partial_{2} c_{\mathcal{Z}}-I\right] P_{\mathcal{U}_{+}^{t}}^{T}} & {\left[\partial_{2} c_{\mathcal{Z}}+I\right] P_{\mathcal{V}_{+}^{t}}^{T}}
\end{array}\right] \\
& \in \mathbb{R}^{\left(m_{1}+|\mathcal{A}|+s_{t}\right) \times\left(n_{t}+\left|\mathcal{U}_{+}^{t}\right|+\left|\mathcal{V}_{+}^{t}\right|\right)}
\end{aligned}
$$

has full row rank $m_{1}+|\mathcal{A}|+s_{t}$. Here all partial derivatives are evaluated at $\left(t, u^{t}+v^{t}\right)$.

Definition 3.5 (MPCC-MFCQ for (I-MPCC), see [11]). We say that the MPCC-MFCQ holds for (I-MPCC) at a feasible point $\left(t, u^{t}, v^{t}\right)$ if

$$
\left[\begin{array}{ccc}
\partial_{1} c_{\mathcal{E}} & \partial_{2} c_{\mathcal{E}} P_{\mathcal{U}_{+}^{t}}^{T} & \partial_{2} c_{\mathcal{E}} P_{\mathcal{V}_{+}^{t}}^{T} \\
\partial_{1} c_{\mathcal{Z}} & {\left[\partial_{2} c_{\mathcal{Z}}-I\right] P_{\mathcal{U}_{+}^{t}}^{T}} & {\left[\partial_{2} c_{\mathcal{Z}}+I\right] P_{\mathcal{V}_{+}^{t}}^{T}}
\end{array}\right] \in \mathbb{R}^{\left(m_{1}+s_{t}\right) \times\left(n_{t}+\left|\mathcal{U}_{+}^{t}\right|+\left|\mathcal{V}_{+}^{t}\right|\right)}
$$

has full row rank $m_{1}+s_{t}$ and if there exists a vector $d \in \mathbb{R}^{n_{t}+\left|\mathcal{U}_{+}^{t}\right|+\left|\mathcal{V}_{+}^{t}\right|}$ such that

$$
\begin{array}{r}
{\left[\begin{array}{ccc}
\partial_{1} c_{\mathcal{E}} & \partial_{2} c_{\mathcal{E}} P_{\mathcal{U}_{+}^{t}}^{T} & \partial_{2} c_{\mathcal{E}} P_{\mathcal{V}_{+}^{t}}^{T} \\
\partial_{1} c_{\mathcal{Z}} & {\left[\partial_{2} c_{\mathcal{Z}}-I\right] P_{\mathcal{U}_{+}^{t}}^{T}} & {\left[\partial_{2} c_{\mathcal{Z}}+I\right] P_{\mathcal{V}_{+}^{t}}^{T}}
\end{array}\right] d=0,} \\
{\left[\begin{array}{lll}
\partial_{1} c_{\mathcal{A}} & \partial_{2} c_{\mathcal{P}} P_{\mathcal{U}_{+}^{t}}^{T} & \partial_{2} c_{\mathcal{A}} P_{\mathcal{V}_{+}^{t}}^{T}
\end{array}\right] d>0 .}
\end{array}
$$

Again all partial derivatives are evaluated at $\left(t, u^{t}+v^{t}\right)$.

As with LIKQ and IDKQ for (I-NLP), MPCC-MFCQ is weaker then MPCC-LICQ for the counterpart MPCC of (I-NLP). The latter fact is well known, and can also be seen easily by rewriting Example 2.6 as the counterpart MPCC and checking the above conditions. 


\subsection{COUNTERPART MPCC FOR THE ABS-NORMAL NLP WITH INEQUALITY SLACKS}

Using the same approach as in the preceding paragraph, we formulate the counterpart MPCC of (E-NLP).

Definition 3.6 (Counterpart MPCC of (E-NLP)). The counterpart MPCC of the abs-normal NLP (E-NLP) reads:

(E-MPCC)

$$
\begin{array}{ll}
\min _{t, w, u^{t}, v^{t}, u^{w}, v^{w}} f(t) \quad \text { s.t. } & c_{\mathcal{E}}\left(t, u^{t}+v^{t}\right)=0 \\
& c_{\mathcal{I}}\left(t, u^{t}+v^{t}\right)-\left(u^{w}+v^{w}\right)=0, \\
& c_{\mathcal{Z}}\left(t, u^{t}+v^{t}\right)-\left(u^{t}-v^{t}\right)=0 \\
& w-\left(u^{w}-v^{w}\right)=0 \\
& 0 \leq u^{t} \perp v^{t} \geq 0 \\
& 0 \leq u^{w} \perp v^{w} \geq 0
\end{array}
$$

where $u^{t}, v^{t} \in \mathbb{R}^{s_{t}}$ and $u^{w}, v^{w} \in \mathbb{R}^{m_{2}}$.

The feasible set of (E-MPCC) is a lifting of $\mathcal{F}_{\text {mpcc }}$ :

$$
\begin{aligned}
\mathcal{F}_{\mathrm{e}-\mathrm{mpcc}} & :=\left\{\begin{array}{l|l}
\left(t, w, u^{t}, v^{t}, u^{w}, v^{w}\right) & \begin{array}{l}
c_{\mathcal{E}}\left(t, u^{t}+v^{t}\right)=0, c_{\mathcal{I}}\left(t, u^{t}+v^{t}\right)=u^{w}+v^{w}, \\
c_{\mathcal{Z}}\left(t, u^{t}+v^{t}\right)=u^{t}-v^{t}, w=u^{w}-v^{w}, \\
0 \leq u^{t} \perp v^{t} \geq 0,0 \leq u^{w} \perp v^{w} \geq 0
\end{array}
\end{array}\right\} \\
& =\left\{\begin{array}{l|l}
\left(t, w, u^{t}, v^{t}, u^{w}, v^{w}\right) & \begin{array}{l}
\left(t, u^{t}, v^{t}\right) \in \mathcal{F}_{\mathrm{mpcc}}, c_{\mathcal{I}}\left(t, u^{t}+v^{t}\right)=u^{w}+v^{w} \\
w=u^{w}-v^{w}, 0 \leq u^{w} \perp v^{w} \geq 0
\end{array}
\end{array}\right\} .
\end{aligned}
$$

Clearly, the homeomorphism between $\mathcal{F}_{\text {mpcc }}$ and $\mathcal{F}_{\text {abs }}$ extends to $\mathcal{F}_{\text {e-mpcc }}$ and $\mathcal{F}_{\text {e-abs }}$

Lemma 3.7. Given an abs-normal NLP (E-NLP) and its counterpart MPCC (E-MPCC), we have a homeomorphism $\bar{\phi}: \mathcal{F}_{\mathrm{e}-\mathrm{mpcc}} \rightarrow \mathcal{F}_{\mathrm{e}-\mathrm{abs}}$ defined as

$$
\begin{aligned}
\bar{\phi}\left(t, w, u^{t}, v^{t}, u^{w}, v^{w}\right) & =\left(t, w, u^{t}-v^{t}, u^{w}-v^{w}\right) \\
\bar{\phi}^{-1}\left(t, w, z^{t}, z^{w}\right) & =\left(t, w,\left[z^{t}\right]^{+},\left[z^{t}\right]^{-},\left[z^{w}\right]^{+},\left[z^{w}\right]^{-}\right)
\end{aligned}
$$

Proof. Obvious.

Lemma 3.8. MPCC-LICQ for (E-MPCC) at a feasible point $y=\left(t, w, u^{t}, v^{t}, u^{w}, v^{w}\right)$ is full row rank of

$$
\begin{aligned}
J_{\text {e-mpcc }}(y) & =\left[\begin{array}{cccccc}
\partial_{1} c_{\mathcal{E}} & 0 & \partial_{2} c_{\mathcal{E}} P_{\mathcal{U}_{+}^{t}}^{T} & \partial_{2} c_{\mathcal{E}} P_{\mathcal{V}_{+}^{t}}^{T} & 0 & 0 \\
\partial_{1} c_{\mathcal{I}} & 0 & \partial_{2} c_{\mathcal{I}} P_{\mathcal{U}_{+}^{t}}^{T} & \partial_{2} c_{\mathcal{I}} P_{\mathcal{V}_{+}^{t}}^{T} & -P_{\mathcal{U}_{+}^{w}}^{T} & -P_{\mathcal{V}_{+}^{w}}^{T} \\
\partial_{1} c_{\mathcal{Z}} & 0 & {\left[\partial_{2} c_{\mathcal{Z}}-I\right] P_{\mathcal{U}_{+}^{t}}^{T}} & {\left[\partial_{2} c_{\mathcal{Z}}+I\right] P_{\mathcal{V}_{+}^{t}}^{T}} & 0 & 0 \\
0 & I & 0 & 0 & -P_{\mathcal{U}_{+}^{w}}^{T} & +P_{\mathcal{V}_{+}^{w}}^{T}
\end{array}\right] \\
& \in \mathbb{R}^{\left(m_{1}+m_{2}+s_{t}+m_{2}\right) \times\left(n_{t}+m_{2}+\left|\mathcal{U}_{+}^{t}\right|+\left|\mathcal{V}_{+}^{t}\right|+\left|\mathcal{U}_{+}^{w}\right|+\left|\mathcal{V}_{+}^{w}\right|\right)},
\end{aligned}
$$

where all partial derivatives are evaluated at $\left(t, u^{t}+v^{t}\right)$.

Proof. We set $x=(t, w), u=\left(u^{t}, u^{w}\right), v=\left(v^{t}, v^{w}\right)$ as well as $\bar{f}(x)=f(t)$,

$$
\bar{c}_{\mathcal{E}}(x, u+v)=\left(\begin{array}{c}
c_{\mathcal{E}}\left(t, u^{t}+v^{t}\right) \\
\left.c_{\mathcal{I}}\left(t, u^{t}+v^{t}\right)-\left(u^{w}+v^{w}\right)\right)
\end{array}\right), \quad \bar{c}_{\mathcal{Z}}(x, u+v)=\left(\begin{array}{c}
c_{\mathcal{Z}}\left(t, u^{t}+v^{t}\right) \\
w
\end{array}\right) .
$$


Then, (E-MPCC) becomes

$(\overline{\mathrm{E}-\mathrm{MPCC}})$

$$
\begin{array}{ll}
\min _{x, u, v} \bar{f}(x) \quad \text { s.t. } \quad & \bar{c}_{\mathcal{E}}(x, u+v)=0, \\
& \bar{c}_{\mathcal{Z}}(x, u+v)-(u-v)=0, \\
& 0 \leq u \perp v \geq 0,
\end{array}
$$

and we can compute the Jacobian from Definition 3.4 using the special structure of (E-MPCC). The resulting matrix has the stated form, except that the last four columns belong to variables $\left(u^{t}, v^{t}, u^{w}, v^{w}\right)$ rather than $(u, v)=\left(u^{t}, u^{w}, v^{t}, v^{w}\right)$.

Like LIKQ for (E-NLP), MPCC-LICQ for (E-MPCC) does not depend on the particular choice of $w$, and like IDKQ for (E-NLP), the concept of MPCC-MFCQ for (E-MPCC) is equivalent to MPCC-LICQ since no inequalities are present besides the complementarities.

\subsection{RELATIONS OF MPCC CONSTRAINT QUALIFICATIONS}

In this paragraph we state the relations of constraint qualifications for the two different formulations introduced in the previous paragraphs. They follow from the results in the previous section and in the two following sections. For an illustration see Figure 1 below. We set $W\left(t, u^{t}, v^{t}\right):=\left\{\left(w, u^{w}, v^{w}\right):|w|=\right.$ $\left.c_{\mathcal{I}}\left(t, u^{t}+v^{t}\right), u^{w}=[w]^{+}, v^{w}=[w]^{-}\right\}$.

Theorem 3.9. MPCC-LICQ for (I-MPCC) holds at $\left(t, u^{t}, v^{t}\right) \in \mathcal{F}_{\text {mpcc }}$ if and only if MPCC-LICQ for (E-MPCC) holds at $\left(t, w, u^{t}, u^{w}, v^{t}, v^{w}\right) \in \mathcal{F}_{\mathrm{e}-\mathrm{mpcc}}$ for any (and hence all) $\left(w, u^{w}, v^{w}\right) \in W\left(t, u^{t}, v^{t}\right)$.

Proof. This follows directly from Theorem 2.11, Theorem 4.1 and Theorem 4.3.

Theorem 3.10. MPCC-MFCQ for (I-MPCC) holds at $\left(t, u^{t}, v^{t}\right) \in \mathcal{F}_{\text {mpcc }}$ if MPCC-MFCQ for (E-MPCC) holds at $\left(t, w, u^{t}, u^{w}, v^{t}, v^{w}\right) \in \mathcal{F}_{\mathrm{e}-\mathrm{mpcc}}$ for any (and hence all) $\left(w, u^{w}, v^{w}\right) \in W\left(t, u^{t}, v^{t}\right)$. The converse is not true.

Proof. This follows directly from Theorem 2.12, Theorem 4.2 and Corollary 4.4.

\section{KINK QUALIFICATIONS AND MPCC CONSTRAINT QUALIFICATIONS}

In this section we have a closer look at relations between abs-normal NLPs and counterpart MPCCs in both formulations.

\subsection{RELATIONS OF GENERAL ABS-NORMAL NLP AND MPCC}

Here we use the variables $x$ and $z$ instead of $t$ and $z^{t}$ to shorten notation because we do not consider inequality slacks. Then the general abs-normal NLP (I-NLP) becomes:

$$
\begin{aligned}
\min _{x, z} f(x) \quad \text { s.t. } & c_{\mathcal{E}}(x,|z|)=0, \\
& c_{\mathcal{I}}(x,|z|) \geq 0, \\
& c_{\mathcal{Z}}(x,|z|)-z=0 .
\end{aligned}
$$

The counterpart MPCC (I-MPCC) reads:

$$
\begin{aligned}
\min _{x, u, v} f(x) \quad \text { s.t. } & c_{\mathcal{E}}(x, u+v)=0, \\
& c_{\mathcal{I}}(x, u+v) \geq 0, \\
& c_{\mathcal{Z}}(x, u+v)-(u-v)=0, \\
& 0 \leq u \perp v \geq 0 .
\end{aligned}
$$


We obtain the following relations of kink qualifications and MPCC constraint qualifications.

Theorem 4.1 (LIKQ $\Longleftrightarrow$ MPCC-LICQ). LIKQ for (I-NLP) at $x \in \mathcal{F}_{\text {abs }}$ is equivalent to MPCC-LICQ for $(\mathrm{I}-\mathrm{MPCC})$ at $(x, u, v)=\left(x,[z(x)]^{+},[z(x)]^{-}\right) \in \mathcal{F}_{\text {mpcc }}$.

Proof. Setting $y:=(x, u+v)$ and $r:=m_{1}+|\mathcal{A}|+s$, MPCC-LICQ for the counterpart MPCC is

$$
\operatorname{rank}\left[\begin{array}{ccc}
\partial_{1} c_{\mathcal{E}}(y) & \partial_{2} c_{\mathcal{E}}(y) P_{\mathcal{U}_{+}}^{T} & \partial_{2} c_{\mathcal{E}}(y) P_{\mathcal{V}_{+}}^{T} \\
\partial_{1} c_{\mathcal{A}}(y) & \partial_{2} c_{\mathcal{A}}(y) P_{\mathcal{U}_{+}}^{T} & \partial_{2} c_{\mathcal{A}}(y) P_{\mathcal{V}_{+}}^{T} \\
\partial_{1} c_{\mathcal{Z}}(y) & {\left[\partial_{2} c_{\mathcal{Z}}(y)-I\right] P_{\mathcal{U}_{+}}^{T}} & {\left[\partial_{2} c_{\mathcal{Z}}(y)+I\right] P_{\mathcal{V}_{+}}^{T}}
\end{array}\right]=r .
$$

By negating the second column and combining it with the third column, this is equivalent to

$$
\operatorname{rank}\left[\begin{array}{cc}
\partial_{1} c_{\mathcal{E}}(y) & -\partial_{2} c_{\mathcal{E}}(y) \Sigma P_{\mathcal{U}_{+} \cup \mathcal{V}_{+}}^{T} \\
\partial_{1} c_{\mathcal{H}}(y) & -\partial_{2} c_{\mathcal{A}}(y) \Sigma P_{\mathcal{U}_{+} \cup \mathcal{V}_{+}}^{T} \\
\partial_{1} c_{\mathcal{Z}}(y) & {\left[I-\partial_{2} c_{\mathcal{Z}}(y) \Sigma\right] P_{\mathcal{U}_{+} \cup \mathcal{V}_{+}}^{T}}
\end{array}\right]=r
$$

and, by non-singularity of $I-\partial_{2} c_{\mathcal{Z}}(y) \Sigma$, to

$$
\operatorname{rank}\left[\begin{array}{cc}
\partial_{1} c_{\mathcal{E}}(y) & -\partial_{2} c_{\mathcal{E}}(y) \Sigma P_{\mathcal{U}_{+} \cup \mathcal{V}_{+}}^{T} \\
\partial_{1} c_{\mathcal{A}}(y) & -\partial_{2} c_{\mathcal{A}}(y) \Sigma P_{\mathcal{U}_{+} \cup \mathcal{V}_{+}}^{T} \\
{\left[I-\partial_{2} c_{\mathcal{Z}}(y) \Sigma\right]^{-1} \partial_{1} c_{\mathcal{Z}}(y)} & P_{\mathcal{U}_{+} \cup \mathcal{V}_{+}}^{T}
\end{array}\right]=r .
$$

Next, we use the third row to eliminate the entries above $P_{\mathcal{U}_{+} \cup \mathcal{V}_{+}}^{T}$ to obtain

$$
\operatorname{rank}\left[\begin{array}{cc}
\partial_{1} c_{\mathcal{E}}(y)+\partial_{2} c_{\mathcal{E}}(y) \Sigma\left[I-\partial_{2} c_{\mathcal{Z}}(y) \Sigma\right]^{-1} \partial_{1} c_{\mathcal{Z}}(y) & 0 \\
\partial_{1} c_{\mathcal{A}}(y)+\partial_{2} c_{\mathcal{A}}(y) \Sigma\left[I-\partial_{2} c_{\mathcal{Z}}(y) \Sigma\right]^{-1} \partial_{1} c_{\mathcal{Z}}(y) & 0 \\
{\left[I-\partial_{2} c_{\mathcal{Z}}(y) \Sigma\right]^{-1} \partial_{1} c_{\mathcal{Z}}(y)} & P_{\mathcal{U}_{+} \cup \mathcal{V}_{+}}^{T}
\end{array}\right]=r,
$$

which we can write with $u+v=|z|=|z(x)|$ as

$$
\operatorname{rank}\left[\begin{array}{cc}
\partial_{x} c_{\mathcal{E}}(x,|z(x)|) & 0 \\
\partial_{x} c_{\mathcal{A}}(x,|z(x)|) & 0 \\
\partial_{x} z(x) & P_{\mathcal{U}_{+} \cup \mathcal{V}_{+}}^{T}
\end{array}\right]=r
$$

Finally, since $\alpha=\mathcal{D}$ is the complement of $\mathcal{U}_{+} \cup \mathcal{V}_{+}$, this is equivalent to

$$
\operatorname{rank}\left[\begin{array}{c}
\partial_{x} c_{\mathcal{E}}(x,|z(x)|) \\
\partial_{x} c_{\mathcal{A}}(x,|z(x)|) \\
{\left[e_{i}^{T} \partial_{x} z(x)\right]_{i \in \alpha}}
\end{array}\right]=m_{1}+|\mathcal{A}|+|\alpha|
$$

which is LIKQ for the abs-normal NLP.

Theorem 4.2 (IDKQ $\Longleftrightarrow$ MPCC-MFCQ). IDKQ for (I-NLP) at $x \in \mathcal{F}_{\text {abs }}$ is equivalent to MPCC-MFCQ for $(\mathrm{I}-\mathrm{MPCC})$ at $(x, u, v)=\left(x,[z(x)]^{+},[z(x)]^{-}\right) \in \mathcal{F}_{\text {mpcc }}$.

Proof. Again with $y:=(x, u+v)$, MPCC-MFCQ for the counterpart MPCC is

1. full row rank of

$$
\left[\begin{array}{ccc}
\partial_{1} c_{\mathcal{E}}(y) & \partial_{2} c_{\mathcal{E}}(y) P_{\mathcal{U}_{+}}^{T} & \partial_{2} c_{\mathcal{E}}(y) P_{\mathcal{V}_{+}}^{T} \\
\partial_{1} c_{\mathcal{Z}}(y) & {\left[\partial_{2} c_{\mathcal{Z}}(y)-I\right] P_{\mathcal{U}_{+}}^{T}} & {\left[\partial_{2} c_{\mathcal{Z}}(y)+I\right] P_{\mathcal{V}_{+}}^{T}}
\end{array}\right] \in \mathbb{R}^{\left(m_{1}+s\right) \times\left(n+\left|\mathcal{U}_{+} \cup \mathcal{V}_{+}\right|\right)} .
$$

As in the proof of Theorem 4.1, this is seen to be full row rank of

$$
\left[\begin{array}{c}
\partial_{x} c_{\mathcal{E}}(x,|z(x)|) \\
{\left[e_{i}^{T} \partial_{x} z(x)\right]_{i \in \alpha}}
\end{array}\right] \in \mathbb{R}^{\left(m_{1}+|\alpha|\right) \times n} .
$$


2. the existence of a vector $d=\left(d_{x}, d_{u}, d_{v}\right) \in \mathbb{R}^{n+\left|\mathcal{U}_{+} \cup \mathcal{V}_{+}\right|}$such that

$$
\begin{array}{r}
{\left[\begin{array}{ccc}
\partial_{1} c_{\mathcal{E}}(y) & \partial_{2} c_{\mathcal{E}}(y) P_{\mathcal{U}_{+}}^{T} & \partial_{2} c_{\mathcal{E}}(y) P_{\mathcal{V}_{+}}^{T} \\
\partial_{1} c_{\mathcal{Z}}(y) & {\left[\partial_{2} c_{\mathcal{Z}}(y)-I\right] P_{\mathcal{U}_{+}}^{T}} & {\left[\partial_{2} c_{\mathcal{Z}}(y)+I\right] P_{\mathcal{V}_{+}}^{T}}
\end{array}\right] d=0,} \\
{\left[\begin{array}{lll}
\partial_{1} c_{\mathcal{A}}(y) & \partial_{2} c_{\mathcal{A}}(y) P_{\mathcal{U}_{+}}^{T} & \partial_{2} c_{\mathcal{A}}(y) P_{\mathcal{V}_{+}}^{T}
\end{array}\right] d>0 .}
\end{array}
$$

We combine $d_{u}$ and $-d_{v}$ to $d_{u v} \in \mathbb{R}^{\left|\mathcal{U}_{+} \cup \mathcal{V}_{+}\right|}$. Then this is equivalent to

$$
\begin{aligned}
& \partial_{1} c_{\mathcal{E}}(y) d_{x}+\partial_{2} c_{\mathcal{E}}(y) \Sigma P_{\mathcal{U}_{+} \cup \mathcal{V}_{+}}^{T} d_{u v}=0, \\
& \partial_{1} c_{\mathcal{Z}}(y) d_{x}-\left[I-\partial_{2} c_{\mathcal{Z}}(y) \Sigma\right] P_{\mathcal{U}_{+} \cup \mathcal{V}_{+}}^{T} d_{u v}=0, \\
& \partial_{1} c_{\mathcal{A}}(y) d_{x}+\partial_{2} c_{\mathcal{A}}(y) \Sigma P_{\mathcal{U}_{+} \cup \mathcal{V}_{+}}^{T} d_{u v}>0 .
\end{aligned}
$$

The second condition can be written as

$$
\left[I-\partial_{2} c_{\mathcal{Z}}(y) \Sigma\right]^{-1} \partial_{1} c_{\mathcal{Z}}(y) d_{x}=P_{\mathcal{U}_{+} \cup \mathcal{V}_{+}}^{T} d_{u v} .
$$

Multiplying this by $P_{\mathcal{D}}^{T}=P_{\alpha}^{T}$ yields

$$
\left[e_{i}^{T}\left[I-\partial_{2} c_{\mathcal{Z}}(y) \Sigma\right]^{-1} \partial_{1} c_{\mathcal{Z}}(y)\right]_{i \in \alpha} d_{x}=\left[e_{i}^{T} \partial_{x} z(x)\right]_{i \in \alpha} d_{x}=0 .
$$

With $u+v=|z|=|z(x)|$, substituting the right-hand side of (4.1) into the first and third condition finally gives

$$
\begin{array}{r}
\partial_{x} c_{\mathcal{E}}(x,|z(x)|) d_{x}=0, \\
{\left[e_{i}^{T} \partial_{x} z(x)\right]_{i \in \alpha} d_{x}=0,} \\
\partial_{x} c_{\mathcal{A}}(x,|z(x)|) d_{x}>0,
\end{array}
$$

which is IDKQ for the abs-normal NLP.

\subsection{RELATIONS OF ABS-NORMAL NLP AND MPCC WITH INEQUALITY SLACKS}

As the reformulation with inequality slacks is just a specialization of the general case, we do without proofs and give remarks where differences occur.

Using the short notation $(\overline{\mathrm{E}-\mathrm{NLP}})$ for (E-NLP) (see proof of Lemma 2.9 ) and similarly $(\overline{\mathrm{E}-\mathrm{MPCC}})$ for the counterpart MPCC (E-MPCC) (see proof of Lemma 3.8), we obtain the same relation between LIKQ and MPCC-LICQ as in the previous paragraph.

Theorem 4.3 (LIKQ $\Longleftrightarrow$ MPCC-LICQ). LIKQ for (E-NLP) at $x \in \mathcal{F}_{\mathrm{e}-\text { abs }}$ is equivalent to MPCC-LICQ for $(\mathrm{E}-\mathrm{MPCC})$ at $(x, u, v)=\left(x,[z(x)]^{+},[z(x)]^{-}\right) \in \mathcal{F}_{\mathrm{e}-\mathrm{mpcc}}$.

Proof. This follows as in the proof of Theorem 4.1.

Note that this directly implies the next result since LIKQ and IDKQ as well as MPCC-LICQ and MPCC-MFCQ coincide in the purely equality constrained setting.

Corollary 4.4 (IDKQ $\Longleftrightarrow$ MPCC-MFCQ). IDKQ for (E-NLP) at $x \in \mathcal{F}_{\mathrm{e}-\text { abs }}$ is equivalent to MPCC-MFCQ for (E-MPCC) at $(x, u, v)=\left(x,[z(x)]^{+},[z(x)]^{-}\right) \in \mathcal{F}_{\mathrm{e}-\mathrm{mpcc}}$. 


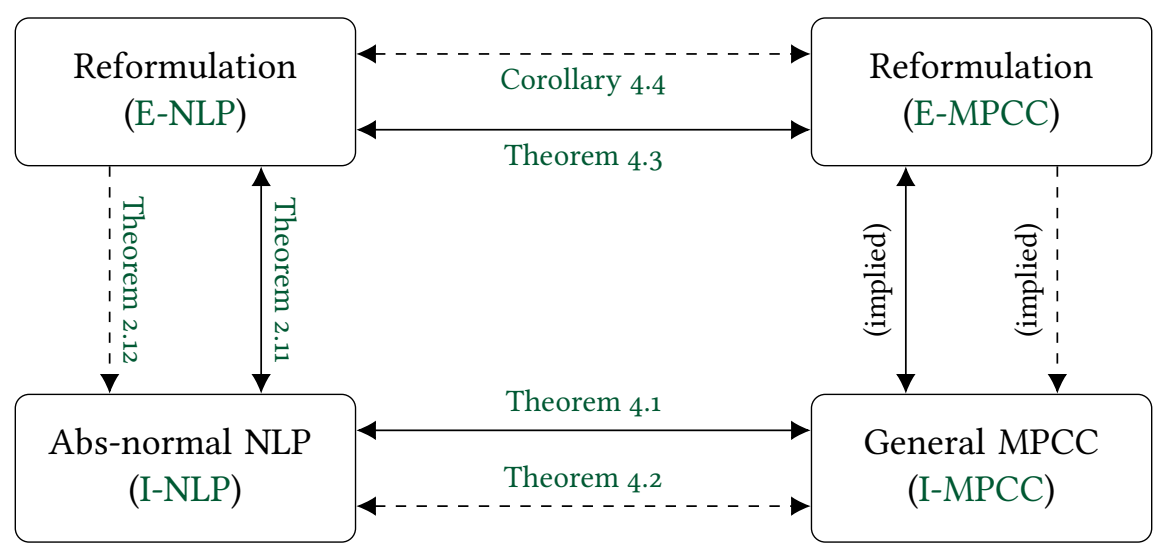

Figure 1: Solid arrows: relations between LIKQ and MPCC-LICQ; dashed arrows: relations between IDKQ and MPCC-MFCQ.

\section{OPTIMALITY CONDITIONS}

In this section we consider first and second order optimality conditions for (I-MPCC) under MPCC-LICQ and for (I-NLP) under LIKQ, respectively, and discuss their relations. Since both regularity conditions are invariant under the slack reformulation by Theorem 2.11 and Theorem 3.9, the results hold also for (E-MPCC) and (E-NLP). Conditions for general MPCCs can be found in the literature; in case of first order conditions for example in [10, 11]. Second order conditions stated in $[10,11]$ however have to be adapted to our different setting. For the abs-normal NLP (E-NLP) we have derived first and second order conditions in [9]. Since LIKQ is preserved under the slack reformulation by Theorem 2.11, we can transfer these results directly to (I-NLP).

\subsection{FIRST ORDER OPTIMALITY CONDITIONS}

In this paragraph, we compare stationarity concepts and first order optimality conditions for (I-MPCC) and (I-NLP). First, we define strong stationarity for (I-MPCC) and state the corresponding first order conditions.

Definition 5.1 (Strong Stationarity, see [10, §3.3], [11, Thm. 2]). A feasible point $y^{*}=\left(t^{*},\left(u^{t}\right)^{*},\left(v^{t}\right)^{*}\right)$ of (I-MPCC) is strongly stationary (S-stationary) if there exist multipliers $\lambda=\left(\lambda_{\mathcal{E}}, \lambda_{I}, \lambda_{\mathcal{Z}}\right)$ and $\mu=\left(\mu_{u}, \mu_{v}\right)$ such that the following conditions are satisfied:

$$
\begin{aligned}
\partial_{y} \mathcal{L}_{\perp}\left(y^{*}, \lambda, \mu\right) & =0, \\
\left(\mu_{u}\right)_{i} \geq 0,\left(\mu_{v}\right)_{i} & \geq 0, \quad i \in \mathcal{D}^{t}\left(t^{*}\right), \\
\left(\mu_{u}\right)_{i} & =0, \quad i \in \mathcal{U}_{+}^{t}\left(t^{*}\right), \\
\left(\mu_{v}\right)_{i} & =0, \quad i \in \mathcal{V}_{+}^{t}\left(t^{*}\right), \\
\lambda_{I} & \geq 0, \\
\lambda_{I}^{T} c_{I}\left(t^{*},\left(u^{t}\right)^{*}+\left(v^{t}\right)^{*}\right) & =0 .
\end{aligned}
$$

Herein, $\mathcal{L}_{\perp}$ is the MPCC-Lagrangian function associated with (I-MPCC):

$$
\begin{aligned}
\mathcal{L}_{\perp}(y, \lambda, \mu):=f(t) & +\lambda_{\mathcal{E}}^{T} c_{\mathcal{E}}\left(t, u^{t}+v^{t}\right)-\lambda_{\mathcal{I}}^{T} c_{\mathcal{I}}\left(t, u^{t}+v^{t}\right) \\
& +\lambda_{\mathcal{Z}}^{T}\left[c_{\mathcal{Z}}\left(t, u^{t}+v^{t}\right)-\left(u^{t}-v^{t}\right)\right]-\mu_{u}^{T} u^{t}-\mu_{v}^{T} v^{t}
\end{aligned}
$$


Theorem 5.2 (First Order Optimality Conditions for (I-MPCC)). Assume that $\left(t^{*},\left(u^{t}\right)^{*},\left(v^{t}\right)^{*}\right)$ is a local minimizer of (I-MPCC) and that MPCC-LICQ holds at $\left(t^{*},\left(u^{t}\right)^{*},\left(v^{t}\right)^{*}\right)$. Then, $\left(t^{*},\left(u^{t}\right)^{*},\left(v^{t}\right)^{*}\right)$ is an S-stationary point.

Proof. The proof is due to $[10, \S 3.3]$ and is presented in [11, Thm. 2] in the form used here.

Now, kink stationarity is defined and the corresponding first order optimality conditions are formulated.

Definition 5.3 (Kink Stationarity, see [9]). A feasible point $\left(t^{*},\left(z^{t}\right)^{*}\right)$ of (I-NLP) is kink stationary if there exist multipliers $\lambda=\left(\lambda_{\mathcal{E}}, \lambda_{I}, \lambda_{\mathcal{Z}}\right)$ such that the following conditions are satisfied:

$$
\begin{aligned}
f^{\prime}\left(t^{*}\right)+\lambda_{\mathcal{E}}^{T} \partial_{1} c_{\mathcal{E}}-\lambda_{I}^{T} \partial_{1} c_{I}+\lambda_{\mathcal{Z}}^{T} \partial_{1} c_{\mathcal{Z}} & =0, & & \\
{\left[\lambda_{\mathcal{E}}^{T} \partial_{2} c_{\mathcal{E}}-\lambda_{I}^{T} \partial_{2} c_{I}+\lambda_{\mathcal{Z}}^{T} \partial_{2} c_{\mathcal{Z}}\right]_{i} } & \geq\left|\left(\lambda_{\mathcal{Z}}\right)_{i}\right|, & & i \in \alpha^{t}\left(t^{*}\right), \\
{\left[\lambda_{\mathcal{E}}^{T} \partial_{2} c_{\mathcal{E}}-\lambda_{I}^{T} \partial_{2} c_{I}+\lambda_{\mathcal{Z}}^{T} \partial_{2} c_{\mathcal{Z}}\right]_{i} } & =\left(\lambda_{\mathcal{Z}}\right)_{i}\left(\sigma^{t}\right)_{i}^{*}, & & i \notin \alpha^{t}\left(t^{*}\right), \\
\lambda_{I} & \geq 0, & & \\
\lambda_{I}^{T} c_{I} & =0 . & &
\end{aligned}
$$

Here, the constraints and the partial derivatives are evaluated at $\left(t^{*},\left|\left(z^{t}\right)^{*}\right|\right)$.

Theorem 5.4 (First Order Conditions for (I-NLP)). Assume that $\left(t^{*},\left(z^{t}\right)^{*}\right)$ is a local minimizer of (I-NLP) and that LIKQ holds at $t^{*}$. Then, $\left(t^{*},\left(z^{t}\right)^{*}\right)$ is a kink stationary point.

Proof. By Theorem 2.11 we may consider the slack reformulation (E-NLP) instead of (I-NLP). In [9, Theorem 5.10], conditions (5.2) were proven for (E-NLP) using a splitting of the switching variables $z$ and the switching constraints $c_{\mathcal{Z}}$. Without the splitting they read:

$$
\begin{array}{rlrl}
\bar{f}^{\prime}\left(x^{*}\right)+\bar{\lambda}_{\mathcal{E}}^{T} \partial_{1} \bar{c}_{\mathcal{E}}\left(x^{*},\left|z^{*}\right|\right)+\bar{\lambda}_{\mathcal{Z}}^{T} \partial_{1} \bar{c}_{\mathcal{Z}}\left(x^{*},\left|z^{*}\right|\right) & =0, & \\
{\left[\bar{\lambda}_{\mathcal{E}}^{T} \partial_{2} \bar{c}_{\mathcal{E}}\left(x^{*},\left|z^{*}\right|\right)+\bar{\lambda}_{\mathcal{Z}}^{T} \partial_{2} \bar{c}_{\mathcal{Z}}\left(x^{*},\left|z^{*}\right|\right)\right]_{i}} & \geq\left|\left(\bar{\lambda}_{\mathcal{Z}}\right)_{i}\right|, & & i \in \alpha\left(x^{*}\right), \\
{\left[\bar{\lambda}_{\mathcal{E}}^{T} \partial_{2} \bar{c}_{\mathcal{E}}\left(x^{*},\left|z^{*}\right|\right)+\bar{\lambda}_{\mathcal{Z}}^{T} \partial_{2} \bar{c}_{\mathcal{Z}}\left(x^{*},\left|z^{*}\right|\right)\right]_{i}} & =\left(\bar{\lambda}_{\mathcal{Z}}\right)_{i} \sigma_{i}^{*}, & & i \notin \alpha\left(x^{*}\right) .
\end{array}
$$

We rewrite these conditions in the original notation of (E-NLP) with $\bar{\lambda}_{\mathcal{E}}=\left(\lambda_{\mathcal{E}},-\lambda_{I}\right)$ and $\bar{\lambda}_{\mathcal{Z}}=$ $\left(\lambda_{\mathcal{Z}}, \lambda_{\mathcal{Z}}^{w}\right)$, where all derivatives are evaluated at $\left(t^{*},\left|\left(z^{t}\right)^{*}\right|\right)$ :

$$
\begin{array}{rlrl}
f^{\prime}\left(t^{*}\right)+\lambda_{\mathcal{E}}^{T} \partial_{1} c_{\mathcal{E}}-\lambda_{\mathcal{I}}^{T} \partial_{1} c_{I}+\lambda_{\mathcal{Z}}^{T} \partial_{1} c_{\mathcal{Z}} & =0, & & \\
\left(\lambda_{\mathcal{Z}}^{w}\right)^{T} & =0, & & \\
{\left[\lambda_{\mathcal{E}}^{T} \partial_{2} c_{\mathcal{E}}-\lambda_{I}^{T} \partial_{2} c_{I}+\lambda_{\mathcal{Z}}^{T} \partial_{2} c_{\mathcal{Z}}\right]_{i}} & \geq\left|\left(\lambda_{\mathcal{Z}}\right)_{i}\right|, & i \in \alpha^{t}\left(t^{*}\right), \\
{\left[\lambda_{\mathcal{E}}^{T} \partial_{2} c_{\mathcal{E}}-\lambda_{I}^{T} \partial_{2} c_{I}+\lambda_{\mathcal{Z}}^{T} \partial_{2} c_{\mathcal{Z}}\right]_{i}} & =\left(\lambda_{\mathcal{Z}}\right)_{i}\left(\sigma^{t}\right)_{i}^{*}, & i \notin \alpha^{t}\left(t^{*}\right), \\
\lambda_{I} & \geq\left|\left(\lambda_{\mathcal{Z}}^{w}\right)_{i}\right|, & i \in \alpha^{w}\left(w^{*}\right), \\
\lambda_{I} & =\left(\lambda_{\mathcal{Z}}^{w}\right)_{i} \sigma_{i}^{*}, & & i \notin \alpha^{w}\left(w^{*}\right) .
\end{array}
$$

The claim follows by eliminating $\lambda_{\mathcal{Z}}^{w}=0$ and noting that $\alpha^{w}\left(w^{*}\right)=\mathcal{A}\left(t^{*}\right)$.

The next theorem shows that the two stationarity concepts coincide.

Theorem 5.5 (S-Stationarity is Kink Stationarity). A feasible point $\left(t^{*},\left(z^{t}\right)^{*}\right)$ of (I-NLP) is kink stationary if and only if $\left(t^{*},\left(u^{t}\right)^{*},\left(v^{t}\right)^{*}\right)=\left(t^{*},\left[z^{t}\left(t^{*}\right)\right]^{+},\left[z^{t}\left(t^{*}\right)\right]^{-}\right)$of (I-MPCC) is S-stationary. 
Proof. Comparison of the stationarity conditions of (I-NLP) and (I-MPCC) shows directly that (5.1e) and (5.2d) as well as (5.1f) and (5.2e) coincide. Thus, we have to compare the remaining conditions (5.1a) to (5.1d) for (I-MPCC) with (5.2a) to (5.2c) for (I-NLP). Condition (5.1a) of (I-MPCC), where all derivatives are evaluated at $\left(t^{*},\left(u^{t}\right)^{*}+\left(v^{t}\right)^{*}\right)$, is

$$
\begin{aligned}
f^{\prime}\left(t^{*}\right)+\lambda_{\mathcal{E}}^{T} \partial_{1} c_{\mathcal{E}}-\lambda_{I}^{T} \partial_{1} c_{I}+\lambda_{\mathcal{Z}}^{T} \partial_{1} c_{\mathcal{Z}} & =0, \\
\lambda_{\mathcal{E}}^{T} \partial_{2} c_{\mathcal{E}}-\lambda_{I}^{T} \partial_{2} c_{I}+\lambda_{\mathcal{Z}}^{T}\left[\partial_{2} c_{\mathcal{Z}}-I\right]-\mu_{u}^{T} & =0, \\
\lambda_{\mathcal{E}}^{T} \partial_{2} c_{\mathcal{E}}-\lambda_{I}^{T} \partial_{2} c_{I}+\lambda_{\mathcal{Z}}^{T}\left[\partial_{2} c_{\mathcal{Z}}+I\right]-\mu_{v}^{T} & =0 .
\end{aligned}
$$

The first condition coincides with (5.2a). We combine the second and the third condition with conditions (5.1b) to (5.1d), yielding

$$
\begin{array}{lll}
{\left[\lambda_{\mathcal{E}}^{T} \partial_{2} c_{\mathcal{E}}-\lambda_{I}^{T} \partial_{2} c_{I}+\lambda_{\mathcal{Z}}^{T} \partial_{2} c_{\mathcal{Z}}\right.} & ]_{i}=+(\lambda \mathcal{Z})_{i}, & i \in \mathcal{U}_{+}^{t}\left(t^{*}\right), \\
{\left[\lambda_{\mathcal{E}}^{T} \partial_{2} c_{\mathcal{E}}-\lambda_{I}^{T} \partial_{2} c_{\mathcal{I}}+\lambda_{\mathcal{Z}}^{T} \partial_{2} c_{\mathcal{Z}}\right.} & ]_{i}=-\left(\lambda_{\mathcal{Z}}\right)_{i}, & i \in \mathcal{V}_{+}^{t}\left(t^{*}\right), \\
{\left[\lambda_{\mathcal{E}}^{T} \partial_{2} c_{\mathcal{E}}-\lambda_{I}^{T} \partial_{2} c_{\mathcal{I}}+\lambda_{\mathcal{Z}}^{T}\left[\partial_{2} c_{\mathcal{Z}} \pm I\right]\right]_{i} \geq 0,} & & i \in \mathcal{D}^{t}\left(t^{*}\right) .
\end{array}
$$

These are precisely conditions (5.2b) and (5.2c) for (I-NLP) by definition of the index sets and of $\sigma^{*}$.

As LIKQ for (I-NLP) is equivalent to MPCC-LICQ for (I-MPCC), the previous theorem provides a different perspective on Theorem 5.4 and Theorem 5.2: one can be obtained from the other directly via Theorem 5.5 and vice versa.

\subsection{SECOND ORDER CONDITIONS}

In this paragraph, we compare second-order conditions for MPCCs and abs-normal NLPs.

First, we formulate them for (I-MPCC). This is based on material from [11] for MPCCs, but some additional assumptions on the Lagrange multipliers need to be made. These are given in the next definition.

Definition 5.6 (MPCC-Strict Complementarity). Consider a strongly stationary point $\left(t^{*},\left(u^{t}\right)^{*},\left(v^{t}\right)^{*}\right)$ with Lagrange multipliers $\left(\lambda^{*}, \mu^{*}\right)$. We say that MPCC-strict complementarity holds if $\lambda_{i}^{*}>0$ for all $i \in \mathcal{A}$ as well as $\left(\mu_{u}^{*}\right)_{i}>0$ and $\left(\mu_{v}^{*}\right)_{i}>0$ for all $i \in \mathcal{D}^{t}$.

We will show in the next lemma that under MPCC-LICQ and MPCC-strict complementarity the critical cone reduces to the nullspace of the Jacobian of the tightened NLP (with columns reordered according to the index sets $\left.\mathcal{U}_{+}^{t}, \mathcal{U}_{0}^{t}, \mathcal{V}_{+}^{t}, \mathcal{V}_{0}^{t}\right)$,

$$
J\left(y^{*}\right)=\left[\begin{array}{ccccc}
\partial_{1} c_{\mathcal{E}} & \partial_{2} c_{\mathcal{E}} P_{\mathcal{U}_{+}^{t}}^{T} & \partial_{2} c_{\mathcal{E}} P_{\mathcal{U}_{0}^{t}}^{T} & \partial_{2} c_{\mathcal{E}} P_{\mathcal{V}_{+}^{t}}^{T} & \partial_{2} c_{\mathcal{E}} P_{\mathcal{V}_{0}^{t}}^{T} \\
\partial_{1} c_{\mathcal{A}} & \partial_{2} c_{\mathcal{H}} P_{\mathcal{U}_{+}^{t}}^{T} & \partial_{2} c_{\mathcal{H}} P_{\mathcal{U}_{0}^{t}}^{T} & \partial_{2} c_{\mathcal{A}} P_{\mathcal{V}_{+}^{t}}^{T} & \partial_{2} c_{\mathcal{A}} P_{\mathcal{V}_{0}^{t}}^{T} \\
\partial_{1} c_{\mathcal{Z}} & {\left[\partial_{2} c_{\mathcal{Z}}-I\right] P_{\mathcal{U}_{+}^{t}}^{T}} & {\left[\partial_{2} c_{\mathcal{Z}}-I\right] P_{\mathcal{U}_{0}^{t}}^{T}} & {\left[\partial_{2} c_{\mathcal{Z}}+I\right] P_{\mathcal{V}_{+}^{t}}^{T}} & {\left[\partial_{2} c_{\mathcal{Z}}+I\right] P_{\mathcal{V}_{0}^{t}}^{T}} \\
0 & 0 & I & 0 & 0 \\
0 & 0 & 0 & 0 & I
\end{array}\right],
$$

as introduced in Section 3.1. Here, all partial derivatives are evaluated at the point $\left(t^{*},\left(u^{t}\right)^{*}+\left(v^{t}\right)^{*}\right)$. It is readily verified that the nullspace of $J\left(y^{*}\right)$ is spanned by the matrix

$$
U_{\mathrm{mpcc}}\left(y^{*}\right)=\left[\begin{array}{c}
I \\
+P_{\mathcal{U}_{+}^{t}}\left(I-\partial_{2} c_{\mathcal{Z}} \Sigma^{t}\right)^{-1} \partial_{1} c_{\mathcal{Z}} \\
0 \\
-P_{\mathcal{V}_{+}^{t}}\left(I-\partial_{2} c_{\mathcal{Z}} \Sigma^{t}\right)^{-1} \partial_{1} c_{\mathcal{Z}} \\
0
\end{array}\right] \tilde{U}\left(y^{*}\right)
$$


where $\tilde{U}\left(y^{*}\right)$ spans the nullspace of

$$
\left[\begin{array}{c}
\partial_{1} c_{\mathcal{E}}+\partial_{2} c_{\mathcal{E}} \Sigma^{t}\left(I-\partial_{2} c_{\mathcal{Z}} \Sigma^{t}\right)^{-1} \partial_{1} c_{\mathcal{Z}} \\
\partial_{1} c_{\mathcal{A}}+\partial_{2} c_{\mathcal{A}} \Sigma^{t}\left(I-\partial_{2} c_{\mathcal{Z}} \Sigma^{t}\right)^{-1} \partial_{1} c_{\mathcal{Z}} \\
{\left[e_{i}^{T}\left(I-\partial_{2} c_{\mathcal{Z}} \Sigma^{t}\right)^{-1} \partial_{1} c_{\mathcal{Z}}\right]_{i \in \mathcal{D}^{t}}}
\end{array}\right] .
$$

Second order necessary and sufficient conditions for a slightly more general class of MPCCs are given in [11, Theorem 7] using the concept of critical directions. We first specialize the definition from [11] to our setting.

Definition 5.7 (Critical Direction). A vector $d=\left(d t, d u^{t}, d v^{t}\right) \in \mathbb{R}^{n_{t}} \times \mathbb{R}^{s_{t}} \times \mathbb{R}^{s_{t}}$ is called a critical direction at a weakly stationary point $y^{*}$ of (I-MPCC) if

$$
\begin{aligned}
& \min \left(d u_{i}^{t}, d v_{i}^{t}\right)=0, \quad i \in \mathcal{D}^{t}, \\
& d u_{i}^{t}=0, \quad i \in \mathcal{V}_{+}^{t}, \\
& d v_{i}^{t}=0, \quad i \in \mathcal{U}_{+}^{t}, \\
& \partial_{1} c_{\mathcal{A}} d u^{t}+\partial_{2} c_{\mathcal{A}}\left(d u^{t}+d v^{t}\right) \geq 0 \text {, } \\
& \partial_{1} c_{\mathcal{E}} d u^{t}+\partial_{2} c_{\mathcal{E}}\left(d u^{t}+d v^{t}\right)=0, \\
& \partial_{1} c_{\mathcal{Z}} d u^{t}+\left[\partial_{2} c_{\mathcal{Z}}-I\right] d u^{t}+\left[\partial_{2} c_{\mathcal{Z}}+I\right] d v^{t}=0 \text {, } \\
& f^{\prime}\left(t^{*}\right) d t=0,
\end{aligned}
$$

where all constraint derivatives are evaluated at $\left(t^{*},\left(u^{t}\right)^{*}+\left(v^{t}\right)^{*}\right)$.

The set of critical directions is just the nullspace of $J\left(y^{*}\right)$ under stronger assumptions.

Lemma 5.8. Assume that MPCC-LICQ and MPCC-strict complementarity hold at an S-stationary point $y^{*}=\left(t^{*},\left(u^{t}\right)^{*},\left(v^{t}\right)^{*}\right)$ of (I-MPCC) with Lagrange multipliers $\left(\lambda^{*}, \mu^{*}\right)$. Then, the set of critical directions is $\operatorname{ker} J\left(y^{*}\right)$.

Proof. First consider a critical direction $d=\left(d t, d u^{t}, d v^{t}\right)$ at the strongly (hence weakly) stationary point $y^{*}$. Then, (5.5e) and (5.5) imply that rows one and three of $J\left(y^{*}\right) d$ vanish, and by (5.1a) and (5.5g) we further have

$$
\begin{aligned}
0= & \partial_{t, u^{t}, v^{t}} \mathcal{L}_{\perp}\left(y^{*}, \lambda^{*}, \mu^{*}\right) d \\
= & f^{\prime}\left(t^{*}\right) d t+\left(\lambda_{\mathcal{E}}^{*}\right)^{T}\left[\partial_{1} c_{\mathcal{E}} d t+\partial_{2} c_{\mathcal{E}}\left(d u^{t}+d v^{t}\right)\right] \\
& -\left(\lambda_{\mathcal{I}}^{*}\right)^{T}\left[\partial_{1} c_{I} d t+\partial_{2} c_{I}\left(d u^{t}+d v^{t}\right)\right] \\
& +\left(\lambda_{\mathcal{Z}}^{*}\right)^{T}\left[\partial_{1} c_{\mathcal{Z}} d t+\left(\partial_{2} c_{\mathcal{Z}}-I\right) d u^{t}+\left(\partial_{2} c_{\mathcal{Z}}+I\right) d v^{t}\right] \\
& -\left(\mu_{u}^{*}\right)^{T} d u^{t}-\left(\mu_{v}^{*}\right)^{T} d v^{t} \\
= & -\left(\lambda_{I}^{*}\right)^{T}\left[\partial_{1} c_{I} d t+\partial_{2} c_{I}\left(d u^{t}+d v^{t}\right)\right]-\left(\mu_{u}^{*}\right)^{T} d u^{t}-\left(\mu_{v}^{*}\right)^{T} d v^{t} .
\end{aligned}
$$

With $\left(\lambda_{I}^{*}\right)^{T} c_{\mathcal{I}}=0(5.1 \mathrm{f}),\left(\mu_{u}^{*}\right)_{i}=0$ for $i \in \mathcal{U}_{+}^{t}(5.1 \mathrm{c}),\left(\mu_{v}^{*}\right)_{i}=0$ for $i \in \mathcal{V}_{+}^{t}(5.1 \mathrm{~d})$, and (5.5b), (5.5c) we obtain $\left(\lambda_{I}^{*}\right)_{i}=0$ for $i \notin \mathcal{A}$ and further

$$
0=\left(\lambda_{\mathcal{A}}^{*}\right)^{T}\left[\partial_{1} c_{\mathcal{A}} d u^{t}+\partial_{2} c_{\mathcal{A}}\left(d u^{t}+d v^{t}\right)\right]+\sum_{i \in \mathcal{D}^{t}}\left[\left(\mu_{u}^{*}\right)_{i} d u_{i}^{t}+\left(\mu_{v}^{*}\right)_{i} d v_{i}^{t}\right]
$$

All factors in this sum of products are nonnegative by (5.1b), (5.1e), (5.5d), and (5.5a), which implies

$$
\begin{aligned}
& 0=\left(\lambda_{\mathcal{A}}^{*}\right)^{T}\left[\partial_{1} c_{\mathcal{P}} d u^{t}+\partial_{2} c_{\mathcal{A}}\left(d u^{t}+d v^{t}\right)\right], \\
& 0=\left(\mu_{u}^{*}\right)_{i} d u_{i}^{t}=\left(\mu_{v}^{*}\right)_{i} d v_{i}^{t}, \quad i \in \mathcal{D}^{t} .
\end{aligned}
$$


Finally, by MPCC-strict complementarity we have

$$
\begin{aligned}
& 0=\partial_{1} c_{\mathcal{A}} d u^{t}+\partial_{2} c_{\mathcal{A}}\left(d u^{t}+d v^{t}\right), \\
& 0=d u_{i}^{t}=d v_{i}^{t}, \quad i \in \mathcal{D}^{t}
\end{aligned}
$$

and $d u_{i}^{t}=0$ for $i \in \mathcal{U}_{0}^{t}$ as well as $d v_{i}^{t}=0$ for $i \in \mathcal{V}_{0}^{t}$ follow since $\mathcal{U}_{0}^{t}=\mathcal{V}_{+}^{t} \cup \mathcal{D}^{t}$ and $\mathcal{V}_{0}^{t}=\mathcal{U}_{+}^{t} \cup \mathcal{D}^{t}$. Thus $d$ is a nullspace vector of $J\left(y^{*}\right)$.

Conversely, given any nullspace vector $d=\left(d t, d u^{t}, d v^{t}\right)$, the first three rows of $J\left(y^{*}\right) d=0$ yield conditions $(5.5 \mathrm{e}),(5.5 \mathrm{~d})$, and $(5.5 \mathrm{f})$, with equality " $=0$ " in case of $(5.5 \mathrm{~d})$. The last two rows yield $d u_{i}^{t}=0$ for $i \in \mathcal{U}_{0}^{t}$ and $d v_{i}^{t}=0$ for $i \in \mathcal{V}_{0}^{t}$, hence (5.5b), (5.5c), and $d u_{i}^{t}=d v_{i}^{t}=0$ for $i \in \mathcal{D}^{t}$ (5.5a). Moreover, we have $\left(\mu_{u}^{*}\right)_{i}=0$ for $i \in \mathcal{U}_{+}^{t}(5.1 \mathrm{c}),\left(\mu_{v}^{*}\right)_{i}=0$ for $i \in \mathcal{V}_{+}^{t}(5.1 \mathrm{~d})$, and $\left(\lambda_{I}^{*}\right)_{i}=0$ for $i \notin \mathcal{A}(5.1 \mathrm{f})$, so that (5.1a) becomes (5.5g):

$$
0=\partial_{t, u^{t}, v^{t}} \mathcal{L}_{\perp}\left(y^{*}, \lambda^{*}, \mu^{*}\right) d=f^{\prime}\left(t^{*}\right) d t
$$

Thus $d$ is a critical direction.

Now we use [11, Theorem 7] to prove second order necessary and sufficient conditions for our setting. Theorem 5.9 (Second Order Necessary Conditions for (I-MPCC)). Assume that $y^{*}=\left(t^{*},\left(u^{t}\right)^{*},\left(v^{t}\right)^{*}\right)$ is a local minimizer of (I-MPCC) and that MPCC-LICQ holds at $y^{*}$. Denote by $\left(\lambda^{*}, \mu^{*}\right)$ the unique Lagrange multiplier vector and assume further that MPCC-strict complementarity holds. Then,

$$
U_{\operatorname{mpcc}}\left(y^{*}\right)^{T} H_{\operatorname{mpcc}}\left(y^{*}, \lambda^{*}\right) U_{\operatorname{mpcc}}\left(y^{*}\right) \geq 0
$$

where $H_{\mathrm{mpcc}}\left(y^{*}, \lambda^{*}\right)=\partial_{y y}^{2} \mathcal{L}_{\perp}\left(y^{*}, \lambda^{*}, \mu^{*}\right)$. (Note that $\partial_{y y}^{2} \mathcal{L}_{\perp}$ does not depend on $\mu^{*}$.)

Proof. The first part of Theorem 7 in [11] asserts that every critical direction $d$ satisfies the inequality $d^{T} H_{\text {mpcc }}\left(y^{*}, \lambda^{*}\right) d \geq 0$ at a local minimizer $y^{*}$ if MPCC-SMFCQ (cf. [10]) holds at $y^{*}$. Since MPCC-LICQ implies MPCC-SMFCQ and the set of critical directions is ker $J\left(y^{*}\right)$ under our stronger assumptions, the claim follows directly from [11, Theorem 7 .

Remark 5.10. Here we have simplified the exposition by making the assumption of MPCC-strict complementarity, so that we can directly rely on [11, Theorem 7]. However, the second order necessary conditions can also be proved without MPCC-strict complementarity by considering branch problems of (I-MPCC). The corresponding approach for (I-NLP) has been taken in [9], so that Theorem 5.12 below does not require strict complementarity.

Theorem 5.11 (Second Order Sufficient Conditions for (I-MPCC)). Assume that $y^{*}=\left(t^{*},\left(u^{t}\right)^{*},\left(v^{t}\right)^{*}\right)$ is strongly stationary for (I-MPCC) with Lagrange multiplier vector $\left(\lambda^{*}, \mu^{*}\right)$ satisfying MPCC-strict complementarity. Assume further that MPCC-LICQ holds at $y^{*}$, and that

$$
U_{\mathrm{mpcc}}\left(y^{*}\right)^{T} H_{\mathrm{mpcc}}\left(y^{*}, \lambda^{*}\right) U_{\mathrm{mpcc}}\left(y^{*}\right)>0 \text {. }
$$

Then, $y^{*}$ is a strict local minimizer of (I-MPCC).

Proof. In the second part of [11, Theorem 7], our assertion is proved under the weaker assumption that $y^{*}$ is strongly stationary and for every critical direction $d \neq 0$ there exists a Lagrange multiplier vector $\left(\lambda^{*}, \mu^{*}\right)$ such that $d^{T} H_{\text {mpcc }}\left(y^{*}, \lambda^{*}\right) d>0$. Under our additional assumptions of MPCC-LICQ and MPCC-strict complementarity, the set of critical directions is spanned by the matrix $U_{\text {mpcc }}\left(y^{*}\right)$, see proof of the previous lemma. Thus, the claim follows directly from [11, Theorem 7]. 
We proceed by formulating second-order conditions for (I-NLP). To this end, we denote by $\left(\alpha^{t}\right)^{c}$ the complement of $\alpha^{t}$, and we need the Lagrangian

$$
\mathcal{L}\left(t,\left|z^{t}\right|, \lambda\right)=f(t)+\lambda_{\mathcal{E}}^{T} c_{\mathcal{E}}\left(t,\left|z^{t}\right|\right)-\lambda_{\mathcal{I}}^{T} c_{\mathcal{I}}\left(t,\left|z^{t}\right|\right)+\lambda_{\mathcal{Z}}^{T}\left(c_{\mathcal{Z}}\left(t,\left|z^{t}\right|\right)-\mathcal{P}_{\left(\alpha^{t}\right) c}^{T} \mathcal{P}_{\left(\alpha^{t}\right)} \Sigma^{t}\left|z^{t}\right|\right)
$$

and the matrix

$$
U_{\mathrm{abs}}(t):=\left[\begin{array}{c}
U(t) \\
{\left[e_{i}^{T} \sum \partial_{t} z^{t}(t) U(t)\right]_{i \notin \alpha^{t}}}
\end{array}\right],
$$

where $U(t)$ spans the nullspace of $J_{\text {abs }}(t)$. We also use the Lagrangian

$$
\begin{aligned}
\overline{\mathcal{L}}(x,|z|, \bar{\lambda})=\bar{f}(x) & +\bar{\lambda}_{\mathcal{E}}^{T} \bar{c}_{\mathcal{E}}(x,|z|)+\bar{\lambda}_{\mathcal{Z}}^{T}\left(\bar{c}_{\mathcal{Z}}(x,|z|)-\mathcal{P}_{\alpha^{c}}^{T} \mathcal{P}_{\alpha^{c}} \Sigma|z|\right), \\
=f(t) & +\lambda_{\mathcal{E}}^{T} c_{\mathcal{E}}\left(t,\left|z^{t}\right|\right)-\lambda_{\mathcal{I}}^{T}\left(c_{\mathcal{I}}\left(t,\left|z^{t}\right|\right)-\left|z^{w}\right|\right) \\
& +\lambda_{\mathcal{Z}}^{T}\left(c_{\mathcal{Z}}\left(t,\left|z^{t}\right|\right)-\mathcal{P}_{\left(\alpha^{t}\right)}^{T} \mathcal{P}_{\left(\alpha^{t}\right) c} \Sigma^{t}\left|z^{t}\right|\right) \\
& +\left(\lambda_{\mathcal{Z}}^{w}\right)^{T}\left(w-\mathcal{P}_{\left(\alpha^{w}\right) c}^{T} \mathcal{P}_{\left(\alpha^{w}\right) c} \Sigma^{w}\left|z^{w}\right|\right) .
\end{aligned}
$$

Theorem 5.12 (Second Order Necessary Conditions for (I-NLP)). Assume that $y^{*}=\left(t^{*},\left(z^{t}\right)^{*}\right)$ is a local minimizer of (I-NLP) and that LIKQ holds at $t^{*}$. Denote by $\lambda^{*}$ the unique Lagrange multiplier and set $\alpha^{t}=\alpha^{t}\left(t^{*}\right)$. Then,

$$
U_{\mathrm{abs}}\left(t^{*}\right)^{T} H_{\mathrm{abs}}\left(y^{*}, \lambda^{*}\right) U_{\mathrm{abs}}\left(t^{*}\right) \geq 0
$$

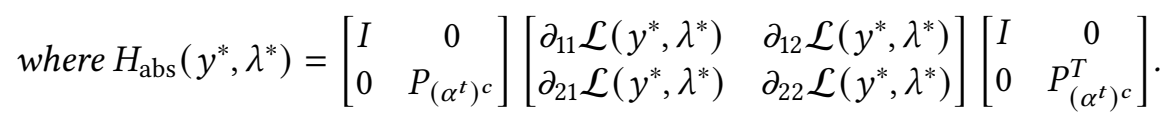

Proof. As in Theorem 5.4, we can consider (E-NLP) instead of (I-NLP) by Theorem 2.11. In [9, Theorem 5.15] the second order necessary conditions for $(\overline{\mathrm{E}-\mathrm{NLP}})$ have been derived using a variable splitting. Without the splitting they read

$$
\bar{U}_{\mathrm{e}-\mathrm{abs}}\left(x^{*}\right)^{T} \bar{H}_{\mathrm{e}-\mathrm{abs}}\left(\bar{y}^{*}, \bar{\lambda}^{*}\right) \bar{U}_{\mathrm{e}-\mathrm{abs}}\left(x^{*}\right) \geq 0
$$

with $\bar{y}^{*}=\left(x^{*}, z^{*}\right), \bar{\lambda}_{\mathcal{E}}=\left(\lambda_{\mathcal{E}},-\lambda_{\mathcal{I}}\right), \bar{\lambda}_{\mathcal{Z}}=\left(\lambda_{\mathcal{Z}}, \lambda_{\mathcal{Z}}^{w}\right)$, and the Hessian

$$
\bar{H}_{\mathrm{e}-\mathrm{abs}}\left(\bar{y}^{*}, \bar{\lambda}^{*}\right)=\left[\begin{array}{cc}
I & 0 \\
0 & P_{\alpha^{c}}
\end{array}\right]\left[\begin{array}{cc}
\partial_{11} \overline{\mathcal{L}}\left(\bar{y}^{*}, \bar{\lambda}^{*}\right) & \partial_{12} \overline{\mathcal{L}}\left(\bar{y}^{*}, \bar{\lambda}^{*}\right) \\
\partial_{21} \overline{\mathcal{L}}\left(\bar{y}^{*}, \bar{\lambda}^{*}\right) & \partial_{22} \overline{\mathcal{L}}\left(\bar{y}^{*}, \bar{\lambda}^{*}\right)
\end{array}\right]\left[\begin{array}{cc}
I & 0 \\
0 & P_{\alpha^{c}}^{T}
\end{array}\right],
$$

where $\alpha^{c}$ is the complement of $\alpha$ and the matrix $\bar{U}_{\mathrm{e}-\text { abs }}$ is defined as

$$
\bar{U}_{\mathrm{e}-\mathrm{abs}}\left(x^{*}\right)=\left[\begin{array}{c}
\bar{U}\left(x^{*}\right) \\
{\left[e_{i}^{T} \Sigma^{*} \partial_{x} z\left(x^{*}\right) \bar{U}\left(x^{*}\right)\right]_{i \notin \alpha}}
\end{array}\right]
$$

with $\bar{U}(x)$ spanning $\operatorname{ker}\left(J_{\mathrm{e}-\mathrm{abs}}(x)\right)$. Using the special structure of $(\overline{\mathrm{E}-\mathrm{NLP}})$ and comparing the derivatives of $\overline{\mathcal{L}}\left(\bar{y}^{*}, \bar{\lambda}^{*}\right)$ and $\mathcal{L}\left(y^{*}, \lambda^{*}\right)$, the Hessian becomes:

$$
\bar{H}_{\mathrm{e}-\mathrm{abs}}\left(\bar{y}^{*}, \bar{\lambda}^{*}\right)=\left[\begin{array}{cccc}
I & 0 & 0 & 0 \\
0 & I & 0 & 0 \\
0 & 0 & P_{\left(\alpha^{t}\right)^{c}} & 0 \\
0 & 0 & 0 & P_{\left(\alpha^{w}\right)^{c}}^{c}
\end{array}\right]\left[\begin{array}{cccc}
\partial_{11} \mathcal{L} & 0 & \partial_{12} \mathcal{L} & 0 \\
0 & 0 & 0 & 0 \\
\partial_{21} \mathcal{L} & 0 & \partial_{22} \mathcal{L} & 0 \\
0 & 0 & 0 & 0
\end{array}\right]\left[\begin{array}{cccc}
I & 0 & 0 & 0 \\
0 & I & 0 & 0 \\
0 & 0 & P_{\left(\alpha^{t}\right)^{c}}^{T} & 0 \\
0 & 0 & 0 & P_{\left(\alpha^{w}\right)^{c}}^{T}
\end{array}\right]
$$

All partial derivatives of $\mathcal{L}$ are evaluated at $\left(y^{*}, \lambda^{*}\right)$. Moreover, $J_{\mathrm{e} \text {-abs }}(x)=J_{\mathrm{e} \text {-abs }}(t, w)$ has the form derived in Lemma 2.9, and thus its nullspace is spanned by

$$
\bar{U}(x)=\left[\begin{array}{c}
U(t) \\
\sum^{w} \partial_{t} c_{I}
\end{array}\right]
$$


where $U(t)$ spans the nullspace of $J_{\mathrm{abs}}(t)$ from Definition 2.4. Using this and $\partial_{t} z^{w}(w)=I$, the matrix $\bar{U}_{\text {e-abs }}$ reads

$$
\bar{U}_{\mathrm{e}-\mathrm{abs}}(x)=\left[\begin{array}{c}
U(t) \\
\sum^{w} \partial_{t} c_{\mathcal{I}} \\
{\left[e_{i}^{T} \Sigma^{t} \partial_{t} z^{t}(t) U(t)\right]_{i \notin \alpha^{t}}} \\
{\left[e_{i}^{T} \partial_{t} c_{I}\right]_{i \notin \alpha^{w}}}
\end{array}\right]
$$

Finally, we have

$$
0 \leq \bar{U}_{\mathrm{e}-\mathrm{abs}}\left(x^{*}\right)^{T} \bar{H}_{\mathrm{e}-\mathrm{abs}}\left(\bar{y}^{*}, \bar{\lambda}^{*}\right) \bar{U}_{\mathrm{e}-\mathrm{abs}}\left(x^{*}\right)=U_{\mathrm{abs}}\left(t^{*}\right)^{T} H_{\mathrm{abs}}\left(y^{*}, \lambda^{*}\right) U_{\mathrm{abs}}\left(t^{*}\right)
$$

with $U_{\text {abs }}(t)$ from (5.6) and

$$
H_{\text {abs }}(y, \lambda)=\left[\begin{array}{cc}
I & 0 \\
0 & P_{\left(\alpha^{t}\right)^{c}}^{c}
\end{array}\right]\left[\begin{array}{cc}
\partial_{11} \mathcal{L}(y, \lambda) & \partial_{12} \mathcal{L}(y, \lambda) \\
\partial_{21} \mathcal{L}(y, \lambda) & \partial_{22} \mathcal{L}(y, \lambda)
\end{array}\right]\left[\begin{array}{cc}
I & 0 \\
0 & P_{\left(\alpha^{t}\right)^{c}}^{T}
\end{array}\right]
$$

This proves the claim.

Theorem 5.13 (Second Order Sufficient Conditions for (I-NLP)). Assume that $y^{*}=\left(t^{*},\left(z^{t}\right)^{*}\right)$ is kink stationary for (I-NLP) with a Lagrange multiplier vector $\lambda^{*}$ that satisfies strict complementarity for $\lambda_{I}^{*}$ and strict normal growth,

$$
\left[\lambda_{\mathcal{E}}^{T} \partial_{2} c_{\mathcal{E}}-\lambda_{I}^{T} \partial_{2} c_{\mathcal{I}}+\lambda_{\mathcal{Z}}^{T} \partial_{2} c_{\mathcal{Z}}\right]_{i}>\left|\left(\lambda_{\mathcal{Z}}\right)_{i}\right|, \quad i \in \alpha^{t}\left(t^{*}\right)
$$

Assume further that LIKQ holds at $t^{*}$, and that

$$
U_{\mathrm{abs}}\left(t^{*}\right)^{T} H_{\mathrm{abs}}\left(y^{*}, \lambda^{*}\right) U_{\mathrm{abs}}\left(t^{*}\right)>0 .
$$

Then, $\left(t^{*},\left(z^{t}\right)^{*}\right)$ is a strict local minimizer of (I-NLP).

Proof. As before we consider the slack reformulation (E-NLP) of (I-NLP). The assumption of strict complementarity for $\lambda_{I}^{*}$ and strict normal growth for (I-NLP) implies strict normal growth for (E-NLP). Moreover, the previous proof shows that the condition

$$
U_{\mathrm{abs}}\left(t^{*}\right)^{T} H_{\mathrm{abs}}\left(y^{*}, \lambda^{*}\right) U_{\mathrm{abs}}\left(t^{*}\right)>0
$$

is equivalent to

$$
\bar{U}_{\mathrm{e}-\mathrm{abs}}\left(x^{*}\right)^{T} \bar{H}_{\mathrm{e}-\mathrm{abs}}\left(\bar{y}^{*}, \bar{\lambda}^{*}\right) \bar{U}_{\mathrm{e}-\mathrm{abs}}\left(x^{*}\right)>0 \text {, }
$$

which can be reformulated using the variable splitting of [9]. Then, [9, Theorem 5.19] can be applied, which gives the assertion.

Theorem 5.14. Assume that $\left(t^{*},\left(z^{t}\right)^{*}\right)$ is kink stationary for (I-NLP) with Lagrange multiplier vector $\lambda^{*}$ such that strict complementarity and strict normal growth are satisfied. Assume further that LIKQ holds at $t^{*}$. Then,

$$
U_{\mathrm{mpcc}}\left(y^{*}\right)^{T} H_{\mathrm{mpcc}}\left(y^{*}, \lambda^{*}\right) U_{\mathrm{mpcc}}\left(y^{*}\right) \geq 0 \Longleftrightarrow U_{\mathrm{abs}}\left(t^{*}\right)^{T} H_{\mathrm{abs}}\left(t^{*},\left(z^{t}\right)^{*}, \lambda^{*}\right) U_{\mathrm{abs}}\left(x^{*}\right) \geq 0,
$$

where $y^{*}=\left(t^{*},\left(u^{t}\right)^{*},\left(v^{t}\right)^{*}\right)=\left(t^{*},\left[\left(z^{t}\right)^{*}\right]^{+},\left[\left(z^{t}\right)^{*}\right]^{-}\right)$. The equivalence holds also with strict inequalities. 
Proof. Using that $u^{*}+v^{*}=\left|\left(z^{t}\right)^{*}\right|$ and $\left(z^{t}\right)^{*}=z^{t}\left(t^{*}\right)$ the matrix $U_{\text {mpcc }}\left(y^{*}\right)$ in (5.3) reads

$$
U_{\mathrm{mpcc}}\left(y^{*}\right)=\left[\begin{array}{c}
I \\
+P_{\mathcal{U}_{+}^{t} \partial_{t} z^{t}\left(t^{*}\right)} \\
0 \\
-P_{\mathcal{V}_{+}^{t}} \partial_{t} z^{t}\left(t^{*}\right) \\
0
\end{array}\right] \tilde{U}\left(y^{*}\right)
$$

with $\tilde{U}\left(y^{*}\right)$ defined in (5.4). The Lagrangians of (I-MPCC) and (I-NLP), respectively, are

$$
\begin{aligned}
\mathcal{L}_{\perp}(y, \lambda) & =f(t)+\lambda_{\mathcal{E}}^{T} c_{\mathcal{E}}\left(t, u^{t}+v^{t}\right)-\lambda_{\mathcal{I}}^{T} c_{\mathcal{I}}\left(t, u^{t}+v^{t}\right)+\lambda_{\mathcal{Z}}^{T}\left[c_{\mathcal{Z}}\left(t, u^{t}+v^{t}\right)-\left(u^{t}-v^{t}\right)\right], \\
\mathcal{L}\left(t,\left|z^{t}\right|, \lambda\right) & =f(t)+\lambda_{\mathcal{E}}^{T} c_{\mathcal{E}}\left(t,\left|z^{t}\right|\right)-\lambda_{\mathcal{I}}^{T} c_{\mathcal{I}}\left(t,\left|z^{t}\right|\right)+\lambda_{\mathcal{Z}}^{T}\left[c_{\mathcal{Z}}\left(t,\left|z^{t}\right|\right)-\mathcal{P}_{\alpha^{c}}^{T} \mathcal{P}_{\alpha^{c}} \Sigma^{t}\left|z^{t}\right|\right] .
\end{aligned}
$$

Thus $U_{\text {mpcc }}\left(y^{*}\right)^{T} H_{\text {mpcc }}\left(y^{*}, \lambda^{*}\right) U_{\text {mpcc }}\left(y^{*}\right)=U_{\text {mpcc }}\left(y^{*}\right)^{T} \nabla_{y y} \mathcal{L}_{\perp}\left(y^{*}, \lambda^{*}\right) U_{\text {mpcc }}\left(y^{*}\right)$ can be written with $\left(z^{t}\right)^{*}=\left(u^{t}\right)^{*}-\left(v^{t}\right)^{*}$ and using that $\tilde{U}\left(y^{*}\right)=U\left(t^{*}\right)$, as

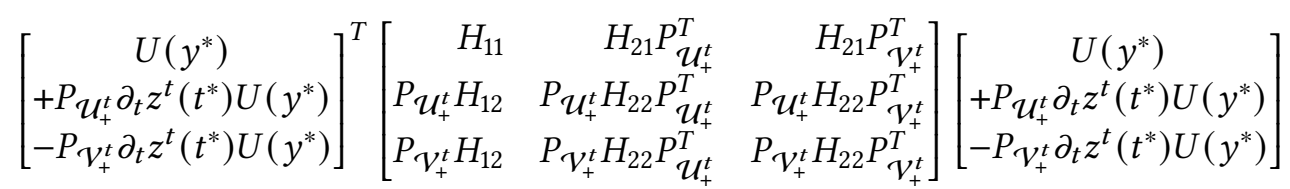

where $H_{i j}:=\partial_{i} \partial_{j} \mathcal{L}\left(t^{*},\left(z^{t}\right)^{*}, \lambda^{*}\right)$. Now, since $\mathcal{U}_{+}^{t} \cup \mathcal{V}_{+}^{t}=\left(\alpha^{t}\right)^{c}$, the left-hand inequality of the claim reads

$$
\left[\begin{array}{c}
U\left(y^{*}\right) \\
P_{\left(\alpha^{t}\right)^{c} \Sigma \partial_{t} z^{t}\left(t^{*}\right) U\left(y^{*}\right)}
\end{array}\right]^{T}\left[\begin{array}{cc}
H_{11} & H_{21} P_{\left(\alpha^{t}\right)^{c}}^{T} \\
P_{\left(\alpha^{t}\right)^{c}} H_{12} & P_{\left(\alpha^{t}\right)^{c}} H_{22} P_{\left(\alpha^{t}\right)^{c}}^{T}
\end{array}\right]\left[\begin{array}{c}
U\left(y^{*}\right) \\
P_{\left(\alpha^{t}\right)^{c} \Sigma \partial_{t} z^{t}\left(t^{*}\right) U\left(y^{*}\right)}
\end{array}\right] \geq 0 .
$$

This is $U_{\mathrm{abs}}\left(t^{*}\right)^{T} H_{\mathrm{abs}}\left(t^{*},\left(z^{t}\right)^{*}, \lambda^{*}\right) U_{\mathrm{abs}}\left(t^{*}\right) \geq 0$.

Note that the previous theorem can be used to transfer the second order conditions for (I-NLP) and (I-MPCC) into each other. This follows from the equivalence of LIKQ and MPCC-LICQ by Theorem 4.1 and from the equivalence of stationarity concepts by Theorem 5.5 .

\section{CONCLUSIONS AND OUTLOOK}

We have shown that general abs-normal NLPs are essentially the same problem class as MPCCs. The two problem classes have corresponding constraint qualifications, stationarity concepts, and optimality conditions of first and second order. We have also shown that the slack reformulation from [9], which is useful to simplify derivations under LIKQ, does not preserve IDKQ and has other subtle drawbacks like non-uniqueness of slack variables. We have not considered counterpart abs-normal NLPs of general MPCCs as in [8]. This would provide a different perspective on the equivalence of the two problem classes but no additional insight. It is hoped that the identities revealed may serve to transfer algorithms for the solution of MPCCs to the young field of abs-normal forms and abs-normal NLP. Vice versa, active signature algorithms for abs-normal forms, such as SALMIN [5], may be applicable to MPCCs. Relations between the two problem classes under weaker constraint qualifications of Abadie type and Guignard type are the subject of part two of this research and are put forward in a companion article. 


\section{REFERENCES}

[1] M. Flegel, Constraint Qualifications and Stationarity Concepts for Mathematical Programs with Equilibrium Constraints, Dissertation, Universität Würzburg, 2005, https://opus.bibliothek.uni-wuerzburg.de/frontdoor/ index/index/year/2005/docld/1068.

[2] A. Griewank, On stable piecewise linearization and generalized algorithmic differentiation, Optimization Methods and Software 28 (2013), 1139-1178, doi:10.1080/10556788.2013.796683.

[3] A. Griewank and A. Walther, First- and second-order optimality conditions for piecewise smooth objective functions, Optimization Methods and Software 31 (2016), 904-930, doi:10.1080/10556788.2016.1189549.

[4] A. Griewank and A. Walther, Characterizing and testing subdifferential regularity for piecewise smooth objective functions, SIAM fournal on Optimization 29 (2019), 1473-1501, doi:10.1137/17m115520x.

[5] A. Griewank and A. Walther, Finite convergence of an active signature method to local minima of piecewise linear functions, Optimization Methods and Software 34 (2019), 1035-1055, doi:10.1080/10556788.2018.1546856.

[6] A. Griewank and A. Walther, Relaxing kink qualifications and proving convergence rates in piecewise smooth optimization, SIAM fournal on Optimization 29 (2019), 262-289, doi:10.1137/17m1157623.

[7] L. C. Hegerhorst-Schultchen, Optimality Conditions for Abs-Normal NLPs, Dissertation, Leibniz Universität Hannover, 2020, doi:10.15488/9867.

[8] L. C. Hegerhorst-Schultchen, C. Kirches, and M. C. Steinbach, On the relation between MPECs and optimization problems in abs-normal form, Optimization Methods and Software 35 (2020), 565-575, doi: $10.1080 / 10556788.2019 .1588268$.

[9] L.C. Hegerhorst-Schultchen and M.C. Steinbach, On first and second order optimality conditions for abs-normal NLP, Optimization 69 (2020), 2629-2656, doi:10.1080/02331934-2019.1626386.

[10] Z. Luo, J. Pang, and D. Ralph, Mathematical Programs with Equilibrium Constraints, Cambridge University Press, 1996.

[11] H. Scheel and S. Scholtes, Mathematical programs with complementarity constraints: stationarity, optimality, and sensitivity, Mathematics of Operations Research 25 (2000), 1-22, doi:10.1287/moor.25.1.1.15213.

[12] J. Ye, Necessary and sufficient optimality conditions for mathematical programs with equilibrium constraints, fournal of Mathematical Analysis and Applications 307 (2005), 350-369, doi:10.1016/j.jmaa.2004.10. 032. 